\title{
Difference of Binding Modes Among Three Ligands to a Receptor mSin3B Corresponding to Their Inhibitory Activities
}

\author{
Tomonori Hayami \\ Osaka University \\ Narutoshi Kamiya \\ University of Hyogo \\ Kota Kasahara \\ Ritsumeikan University \\ Takeshi Kawabata \\ Osaka University \\ Jun-ichi Kurita \\ Yokohama City University \\ Yoshifumi Fukunishi \\ National Institute of Advanced Industrial Science and Technology \\ Yoshifumi Nishimura \\ Yokohama City University \\ Haruki Nakamura \\ Osaka University \\ Junichi Higo ( $\nabla$ higo@protein.osaka-u.ac.jp ) \\ University of Hyogo
}

\section{Research Article}

Keywords: REST/NRSF, YN3, hydrophobic, amino-acid

Posted Date: January 7th, 2021

DOI: https://doi.org/10.21203/rs.3.rs-139177/v1

License: (c) (1) This work is licensed under a Creative Commons Attribution 4.0 International License. Read Full License

Version of Record: A version of this preprint was published at Scientific Reports on March 17th, 2021. See the published version at https://doi.org/10.1038/s41598-021-85612-9. 



\title{
Difference of binding modes among three ligands to a receptor $\mathrm{mSin} 3 \mathrm{~B}$ corresponding to their inhibitory activities
}

\author{
Tomonori Hayami $^{1}$, Narutoshi Kamiya ${ }^{2}$, Kota Kasahara ${ }^{3}$, Takeshi Kawabata ${ }^{1}$, \\ Jun-ichi Kurita ${ }^{4}$, Yoshifumi Fukunishi ${ }^{5}$, Yoshifumi Nishimura ${ }^{4,6}$, Haruki \\ Nakamura $^{1}$, Junichi Higo ${ }^{1,2, *}$ \\ ${ }^{1}$ Institute for Protein Research, Osaka University, 3-2 Yamada-oka, Suita, Osaka 565- \\ 0871, Japan \\ ${ }^{2}$ Graduate School of Simulation Studies, University of Hyogo, 7-1-28 Minatojima \\ Minamimachi, Chuo-ku, Kobe, Hyogo 650-0047, Japan \\ ${ }^{3}$ College of Life Sciences, Ritsumeikan University, 1-1-1 Noji-higashi, Kusatsu, Shiga \\ 525-8577, Japan \\ ${ }^{4}$ Graduate School of Medical Life Science, Yokohama City University, 1-7-29 Suehiro- \\ cho, Tsurumi-ku, Yokohama, 230-0045, Japan \\ ${ }^{5}$ Cellular and Molecular Biotechnology Research Institute, National Institute of \\ Advanced Industrial Science and Technology (AIST), 2-3-26, Aomi, Koto-ku, Tokyo, \\ 135-0064, Japan \\ ${ }^{6}$ Graduate School of Integrated Sciences for Life, Hiroshima University, 1-4-4 \\ Kagamiyama, Higashi-Hiroshima 739-8258, Japan
}

*Correspondence and requests for materials should be addressed to J.H. (e-mail: higo@protein.osaka-u.ac.jp) and K.K. (e-mail: ktkshr@fc.ritsumei.ac.jp)

\begin{abstract}
A preceding experiment suggested that a compound, which inhibits binding of the REST/NRSF segment to the cleft of a receptor protein $\mathrm{mSin} 3 \mathrm{~B}$, can be a potential drug candidate to ameliorate many neuropathies. We have recently developed an enhanced conformational sampling method, genetic-algorithm-guided multi-dimensional virtualsystem-coupled canonical molecular dynamics, and in the present study, applied it to three systems consisting of mSin3B and one of three compounds, sertraline, YN3, and acitretin. Other preceding experiments showed that only sertraline inhibits the binding of REST/NRSF to mSin3B. The current simulation study produced the spatial distribution of the compounds around $\mathrm{mSin} 3 \mathrm{~B}$, and showed that sertraline and $\mathrm{YN} 3$ bound to the cleft of mSin3B with a high propensity, although acitretin did not. Further analyses of the simulation data indicated that only the sertraline-mSin3B complex
\end{abstract}


produced a hydrophobic core similar to that observed in the molecular interface of the REST/NRSF-mSin3B complex: An aromatic ring of sertraline sunk deeply in the mSin3B's cleft forming a hydrophobic core contacting to hydrophobic amino-acid residues located at the bottom of the cleft. The present study proposes a step to design a compound that inhibits competitively the binding of a ligand to its receptor.

\section{Introduction}

Neural restrictive silencer factor (NRSF), which is also known as Repressor-element 1 silencing transcription factor (REST) $)^{1,2}$, is a fundamental repressor, which binds to repressor-element 1 (re1) or neural restrictive silencer element (nrse) of many neuronal genes $^{3,4}$. Importantly, overexpression of REST/NRSF or dysregulation of its cellular expression pattern is related to many neuropathies: Medulloblastoma ${ }^{5,6}$, malignant pediatric brain tumor ${ }^{7}$, glioblastoma ${ }^{8,9}$, Huntington's disease ${ }^{10-13}$, neuropathic pain ${ }^{14,15}$, Parkinson's disease ${ }^{16}$, autism ${ }^{17}$, and fibromyalgia ${ }^{18}$.

REST/NRSF mediates transcriptional repression recruiting two corepressor complexes: REST/NRSF binds to mSin3 at its N-terminus and to CoREST plus the histone H3K9 methyltransferase G9a at its C-terminus ${ }^{19}$. The mSin3 complex, which contains two histone deacetylases HDAC1 and HDAC2 $2^{20}$, was implicated as an important epigenetic regulator in cancer ${ }^{21}$. The corepressor $\mathrm{mSin} 3 \mathrm{~B}$, an isoform of $\mathrm{mSin} 3$, consists of four paired amphipathic helix domains (PAH1-PAH4) connected by linkers among the domains, and an intrinsically disordered region of REST/NRSF binds to the cleft of PAH1 of $m \sin 3 B^{22}$.

Interestingly, an NMR experiment has shown that the disordered regions of REST/NRSF folds into a helix when binding to the hydrophobic cleft of the PAH1 domain $^{22}$ (coupled folding and binding ${ }^{23-25}$ ). See Figure S1 of Supplementary Information for the PAH1 structure and the cleft position in the domain. A microscopic mechanism for the coupled folding and binding of this system was elucidated by our earlier computational study ${ }^{26}$. The NMR work provided a useful strategy for drug discovery: A compound that inhibits the binding of REST/NRSF to the PAH1 cleft of $\mathrm{mSin} 3 \mathrm{~B}$ can be a potential drug candidate to ameliorate the neuropathies ${ }^{18,27-30}$, and many compounds have been examined ${ }^{18,22,31}$.

In fact, Ueda et al. analyzed the NMR complex structure of REST/NRST and the PAH1 domain of $\mathrm{mSin} 3 \mathrm{~B}$, and proposed a compound $\mathrm{mS}-11$ to inhibit the binding of REST/NRSF to the PAH1 domain ${ }^{18}$. This compound mimics the helical structure of a four-residue segment (Leu46-Ile47-Met48-Leu49) of REST/NRSF in the bound form, 
and importantly, this compound inhibited actually the binding of REST/NRST to the PAH1 domain. We call this four-residue segment a LIML sequence in this study.

Recently, we developed a generalized ensemble method, multi-dimensional virtual-system coupled molecular dynamics (mD-VcMD) simulation ${ }^{32,33}$. This method enhances conformational sampling of biomolecules in an explicit solvent: By introducing multiple reaction coordinates (RCs) in the molecular system, the conformational motions of the molecules are enhanced with controlling the values of the multiple RCs. The search region in the RC space is expanded through iterative simulation. Importantly, a thermodynamic weight is assigned to each of the sampled conformations, and various thermodynamic quantities of the system are computed from the weighted snapshots. Then, we extended $\mathrm{mD}-\mathrm{VcMD}$ by using a genetic algorithm and named the method as a geneticalgorithm-guided mD-VcMD (GA-guided mD-VcMD) simulation ${ }^{34,35}$ where the genetic algorithm supports expansion of the search range effectively. We have shown that the sampling efficiency of the GA-guided $\mathrm{mD}-\mathrm{VcMD}$ is significantly higher than that of the original $\mathrm{mD}-\mathrm{VcMD}^{34}$.

In the present study, we investigate the spatial distribution of three compounds sertraline, YN3, and acitretin, respectively, around the PAH1 domain of $\mathrm{mSin} 3 \mathrm{~B}$, which are obtained from the GA-guided $\mathrm{mD}-\mathrm{VcMD}$ simulation of the system. The chemical structures of the three compounds are shown in Supplementary Figures S2-S4. We note that preceding experiments on sertraline and YN3 have shown that only sertraline exhibited medulloblastoma cell growth inhibitory activity, although both compounds bound to the PAH1 domain of $\mathrm{mSin} 3 \mathrm{~B}^{31}$. Another preceding experiment has shown that no inhibitory activities were detected for acitretin whereas it also binds to the PAH1 domain (personal communication with Nishimura). We show that the spatial distribution of the three compounds from the simulations rationally explains why only sertraline exhibited the inhibitory activity. Based on these computational results, we discuss a strategy to develop a drug candidate.

\section{Materials and Methods}

In this study, we denote the PAH1 domain of mSin3B simply as "mSin3B" for convenience. Besides, a system composed of sertraline and $\mathrm{mSin} 3 \mathrm{~B}$ is referred to as a "sertraline-mSin3B" system even though the two molecules are apart to each other during the simulation. Similarly, a system of YN3 and mSin3B is done to as a "YN3-mSin3B" system, and that of acitretin and $\mathrm{mSin} 3 \mathrm{~B}$ as a "acitretin-mSin3B" system.

\section{Three Reaction Coordinates.}


First, we introduced multiple RCs in the system, where an RC was defined by the distance between centers of mass of two atom groups. Supplementary Section 1 presents a detailed method to set an RC in the system, and Supplementary Figure S5 shows schematically the RC set in a system. For each the of ligand-receptor systems, we introduced three RCs, denoted as $\lambda^{(h)}(h=\alpha, \beta, \gamma)$. We briefly explain here the RCs as follows: The two atom groups $G_{\alpha}^{A}$ (red-colored segments in Supplementary Figures S2c, S3c, and S4c) and $G_{\alpha}^{B}$ (blue-colored segments) define the first RC $\lambda^{(\alpha)}$. We can imagine readily that the move of $\lambda^{(\alpha)}$ opens/closes the cleft. Atom groups $G_{\beta}^{A}$ and $G_{\gamma}^{A}$ are respectively green-colored and cyan-colored segments in $\mathrm{mSin} 3 \mathrm{~B}$, and $G_{\beta}^{B}$ and $G_{\gamma}^{B}$ are purple-colored and orangecolored portions in the ligand (Supplementary Figures S2b, S3b, and S4b). The moves of $\lambda^{(\beta)}$ (distance between $G_{\beta}^{A}$ and $G_{\beta}^{B}$ ) and $\lambda^{(\gamma)}$ (distance between $G_{\gamma}^{A}$ and $G_{\gamma}^{B}$ ) control the ligand approaching/departing to $\mathrm{mSin} 3 \mathrm{~B}$. When $\lambda^{(\beta)}$ increases with decreasing $\lambda^{(\gamma)}$ or when $\lambda^{(\beta)}$ decreases with increasing $\lambda^{(\gamma)}$, the ligand rotates. Supplementary Figure S6 illustrate schematically that the system moves according to the variations of RCs in the RC space.

We note that the selection of RCs can be arbitrary in theory if a very long simulation is possible. However, the selection is essentially important to raise the efficiency in an actual simulation. Detailed information for the atom groups is given in Supplementary Table S1.

To study molecular binding extensively, the multi-dimensional $\mathrm{RC}$ region should involve both the unbound and bound conformations. For this purpose, we set the variable ranges for the three RCs wide enough (Supplementary Table S2). The term "multi-dimensional (mD)" means three-dimensional (3D) in this study, whereas the current method is applicable to any dimensional $\mathrm{RC}$ space.

\section{Initial Conformations of Simulation.}

After setting the RCs above, the initial conformation of simulation was generated. First, the tertiary structure of the receptor $\mathrm{mSin} 3 \mathrm{~B}$ (PAH1 domain) was taken from the PDBj site (https://pdbj.org/) (PDB ID: 2CZY), in which the receptor binds to the REST/NRSF fragment. After removing REST/NRSF from the complex, we introduced a ligand (sertraline, $\mathrm{YN} 3$, or acitretin) near $\mathrm{mSin} 3 \mathrm{~B}$. As explained later, we randomized the position of the ligand to generate the initial conformations of the GA-guided $\mathrm{mD}-\mathrm{VcMD}$ simulation, where the ligand was distant from the binding cleft of $\mathrm{mSin} 3 \mathrm{~B}$.

Next, we put the two molecules (ligand and mSin3B) generated above in a periodic boundary box (size is $70.0 \AA^{3}$ ) filled by water molecules, and removed water molecules that overlapped to mSin3B or the ligand. Then $\mathrm{Na}^{+}$and $\mathrm{Cl}^{-}$ions were 
introduced with randomly replacing water molecules by ions. The number of ions was set to a physiological ionic concentration with neutralizing the net charge of the whole system to zero. The resultant sertraline-mSin3B system consists of 33,543 atoms $(1,200$ atoms for $\mathrm{mSin} 3 \mathrm{~B}, 38$ atoms for sertraline, 10,749 water molecules, $29 \mathrm{Na}^{+}, 29 \mathrm{Cl}^{-}$), the YN3-mSin3B system does of 33,533 atoms (1,200 atoms for $\mathrm{mSin} 3 \mathrm{~B}, 40$ atoms for YN3, 10,745 water molecules, $29 \mathrm{Na}^{+}, 29 \mathrm{Cl}^{-}$), and the acitretin-mSin3B system does of 33,525 atoms $\left(1,200\right.$ atoms for $\mathrm{mSin} 3 \mathrm{~B}, 50$ atoms for acitretin, 10,739 water molecules, $29 \mathrm{Na}^{+}$, $29 \mathrm{Cl}^{-}$). After a short energy minimization, a short constant-volume and constanttemperature $(300 \mathrm{~K})$ simulation (NVT simulation) was performed. Then, a constantpressure $(1 \mathrm{~atm})$ and constant-temperature $(300 \mathrm{~K})$ simulation (NPT simulation) was performed to relaxed the box size. The resultant box size was $68.920^{3} \AA^{3}, 68.909^{3}$, and $68.909^{3}$ for the sertraline-mSin3B, YN3-mSin3B, and acitretin-mSin3B systems, respectively.

Whereas the PAH1 domain is linked to the PAH2 domain by a long flexible linker in a real cell, only the PAH1 domain was computed in our simulation, and the interdomain linker was treated as the C-terminal tail. This tail might be inserted into the binding cleft of PAH1 domain incidentally during the simulation. It is likely that the incidental insertion of the inter-domain linker into the cleft does not happen if the PAH1 and PAH domains are connected by the linker. Thus, to prevent this incidental and artificial event, we applied weak restraints to the C-terminal tail (see Supplementary Section 2). By the restraints, the C-terminal did not move to the binding cleft, and fluctuated around the initial conformation (NMR structure; PDB ID: 2CZY) during the simulation.

To generate the initial conformations of simulation, where the ligand is distant from the binding cleft of $\mathrm{mSin} 3 \mathrm{~B}$, we applied interactions between $\mathrm{mSin} 3 \mathrm{~B}$ and the ligand so that the RCs fall in the following ranges: $15 \AA<\lambda^{(\alpha)}<16 \AA, 24 \AA<\lambda^{(\beta)}<25 \AA$, and $24 \AA<\lambda^{(\gamma)}<25 \AA$. Then, with applying these interactions we performed 256 runs starting from the last snapshot of the NPT simulation done above. Supplementary Figures S7-S9 display some of the last conformations picked randomly from those 256 runs for the three systems. We used those 256 conformations for the initial conformation of GAguided $\mathrm{mD}-\mathrm{VcMD}$. Apparently, the ligand in these conformations was distant from the cleft of $\mathrm{mSin} 3 \mathrm{~B}$ (i.e., REST/NRSF position). These figures also display the REST/NRSF fragment binding to the cleft of mSin3B (PDB ID: 2CZY), whereas REST/NRSF did not exist in the current simulation.

\section{The GA-guided mD-VcMD.}


Methodological details for GA-guided mD-VcMD are explained elsewhere ${ }^{34}$. Here, we explain the outline of GA-guided $\mathrm{mD}-\mathrm{VcMD}$ and resultant quantities. This method controls the system's motions by modulating the three $\operatorname{RCs} \lambda^{(h)}(h=\alpha, \beta, \gamma)$. Supplementary Figure S6b presents schematically a distribution of the system's conformation in the 3D RC space resulted from the moves of $\lambda^{(h)}$.

The outline of the method is as follows: First, the entire 3D RC space is divided into many small zones. Then, the GA-guided $\mathrm{mD}-\mathrm{VcMD}$ simulation provides a conformational distribution function $Q_{\text {cano }}\left(\lambda^{(\alpha)}, \lambda^{(\beta)}, \lambda^{(\gamma)}\right)$ of the system, where $Q_{\text {cano }}\left(\lambda^{(\alpha)}, \lambda^{(\beta)}, \lambda^{(\gamma)}\right)$ is the probability of existence at position $\left[\lambda^{(\alpha)}, \lambda^{(\beta)}, \lambda^{(\gamma)}\right]$ in the 3D RC space constructed by $\lambda^{(\alpha)}, \lambda^{(\beta)}$, and $\lambda^{(\gamma)}$. Because the conformational space of a biological molecular system is wide, the GA-guided $\mathrm{mD}-\mathrm{VcMD}$ is executed via iterative simulations, during which the sampled $\mathrm{RC}$ region is expanded. The simulation is terminated when $Q_{\text {cano }}\left(\lambda^{(\alpha)}, \lambda^{(\beta)}, \lambda^{(\gamma)}\right)$ has converged. We discuss the convergence quantitatively later. After the convergence, a thermodynamic weight is assigned to each of stored snapshots using $Q_{\text {cano }}\left(\lambda^{(\alpha)}, \lambda^{(\beta)}, \lambda^{(\gamma)}\right)$, and the ensemble of the snapshots can be regarded as a thermally equilibrated conformational ensemble (canonical ensemble) ${ }^{34}$. If the GA-guided $\mathrm{mD}-\mathrm{VcMD}$ simulation is done at temperature $T$, then the canonical ensemble at $T$ is obtained.

We performed 256 runs for an iteration in the present study to raise the sampling efficiency further. A simple integration of the 256 trajectories can be regarded as a long single trajectory ${ }^{36,37}$. When the $M$-th iteration is finished, we have snapshots stored from the 1 st to $\mathrm{M}$-th iterations. The 256 initial conformations for the $(M+1)$-th iteration were selected from those stored snapshots so that the conformations distributed as even as possible in the 3D-RC space. On the other hand, when an RC region was sampled poorly, we prepared the initial conformations around the poorly sampled region ${ }^{34}$. Because we obtained the canonical ensemble at $300 \mathrm{~K}$ as a result of the GA-guided $\mathrm{mD}-\mathrm{VcMD}$, we calculated the distribution function of various quantities in equilibrium at $300 \mathrm{~K}$.

\section{Simulations.}

The actual parameter values for the GA-guised $\mathrm{mD}-\mathrm{VcMD}$ are presented in Supplementary Section 3. The simulation was performed using a computer program omegagene/myPresto ${ }^{38}$ with the following condition: $\mathrm{SHAKE}^{39}$ to fix the covalent-bond lengths related to hydrogen atoms, the Berendsen thermostat to control temperature ${ }^{40}$, the zero-dipole summation method ${ }^{41-43}$ to compute accurately and quickly the long-range electrostatic interactions, a time-step of $2 \mathrm{fs}(\Delta t=2 \mathrm{fs})$, and simulation temperature of $300 \mathrm{~K}$. The Berendsen thermostat produces an ensemble that can approximate a canonical 
distribution for a many-atom system, whereas it generates a non-physical distribution for a small system ${ }^{44}$. To compute the original potential energy of the system, the Amber hybrid force fields (mixture parameter $w=0.75)^{45}$ was used for $\mathrm{mSin} 3 \mathrm{~B}$, the TIP3P potential model for water molecules ${ }^{46}$, and the Joung-Cheatham model for chloride and sodium ions ${ }^{47}$.

The force fields for the sertraline, YN3, and acitretin were set as follows: First, the atomic partial charges were derived by quantum chemical calculations using Gaussian $03^{48}$ at the HF/6-31G* level, followed by RESP fitting ${ }^{49}$. Then, those partial charges were incorporated into a GAFF (general amber force field) force-field file ${ }^{50}$. GAFF was designed to be compatible with conventional AMBER force-fields. The Amber hybrid force fields currently used for $\mathrm{mSin} 3 \mathrm{~B}$ was generated by ourselves with mixing Amber parm $94^{51}$ and parm $96^{52}$ force fields to treat both helical and stranded polypeptides $^{45}$, and the difference between parm94 and parm96 exists only in the dihedral energy parameters. Therefore, the inter-molecular interaction energy between $\mathrm{mSin} 3 \mathrm{~B}$ and the ligands is invariant mechanically among the parm94, parm96, and hybrid force fields.

\section{Spatial density of a compound around mSin3B.}

As mentioned above, the GA-guided VcMD simulation produces a conformational ensemble, where a thermodynamic weight at $300 \mathrm{~K}$ is assigned to each constituent conformation. Thus, we can calculate a spatial distribution function of any structural quantity from the ensemble. In this study, we compute the spatial density $\rho_{C G}^{(s)}(\boldsymbol{r})$, which is the probability of detecting the geometrical center (GC) of the ligand in the vicinity of a three-dimensional position $\boldsymbol{r}=[\boldsymbol{x}, \boldsymbol{y}, \boldsymbol{z}]$ in the real space, where the superscript $s$ is the system specifier: $s=$ sertraline-mSin3B, YN3-mSin3B, or acitretin-mSin3B. The detailed computational procedure to calculate $\rho_{C G}^{(s)}(\boldsymbol{r})$ is explained in Supplementary Section 4. Other spatial density functions were calculated with the same manner: See Supplementary Eq. S4.

\section{Results}

\section{Distribution of the system's conformation in 3D RC space.}

We repeated 14, 13, and 27 iterations for the sertraline-mSin3B, YN3-mSin3B, and acitretin-mSin3B systems, respectively. For all of the three systems, an iteration was composed of 256 runs. Each run was performed for $3 \times 10^{6}$ steps (6 ns; time step $\Delta t=$ $2 \mathrm{fs})$. Thus, the total simulation length was $21.504 \mu \mathrm{s}(=14 \times 256 \times 6 \mathrm{~ns}), 19.968 \mu \mathrm{s}$ $(=13 \times 256 \times 6 \mathrm{~ns})$, and $41.472 \mu \mathrm{s}(=27 \times 256 \times 6 \mathrm{~ns})$ for the sertraline-mSin3B, YN3- 
$\mathrm{mSin} 3 \mathrm{~B}$, and acitretin-mSin3B systems, respectively. A snapshot was stored every $1 \times 10^{5}$ steps (200 ps), yielding 107,520, 99,840, and 207,360 snapshots, for the sertraline-mSin3B, YN3-mSin3B, and acitretin-mSin3B systems, respectively.

Figure 1 demonstrates the conformational distributions of the three systems in the 3D RC space, where the density is normalized so that the largest density is set to 1.0. We checked the convergence of distribution during the iterations. The method to check the convergence is given in Supplementary Section 5. Supplementary Figures S10-S12 indicate that the regions with $Q_{\text {cano }}\left(\lambda^{(\alpha)}, \lambda^{(\beta)}, \lambda^{(\gamma)}\right)>0.001$ are converged well. Although Figure 1 is basically important to show that the sampling covered a wide conformational space, this figure is not useful for understanding the ligand's distribution around $\mathrm{mSin} 3 \mathrm{~B}$. In the next section, we analyze the ligand's distribution using the canonical conformational ensemble.

\section{Fig. 1}

(a)

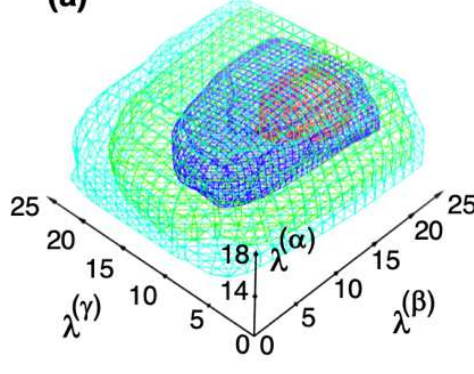

(b)

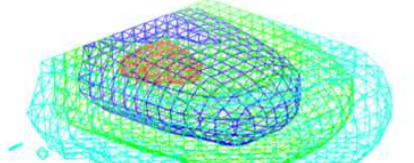

(c)

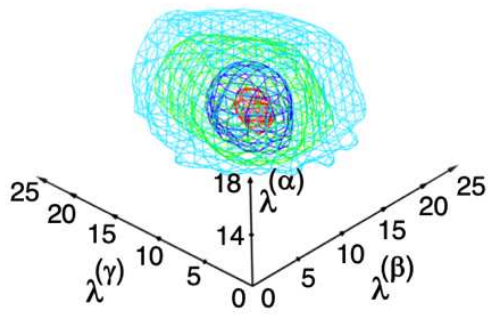

$$
\longrightarrow Q(\lambda)=0.5 \quad \longrightarrow Q(\lambda)=0.1 \quad \longrightarrow Q(\lambda)=0.01 \quad Q(\lambda)=0.001
$$

Figure 1. Density $Q_{\text {cano }}\left(\lambda^{(\alpha)}, \lambda^{(\beta)}, \lambda^{(\gamma)}\right)$ of (a) the sertraline-mSin3B, (b) YN3-mSin3B, and (c) acitretin$\mathrm{mSin} 3 \mathrm{~B}$ systems in the 3D-RC space. In GA-guided $\mathrm{mD}-\mathrm{VcMD}$, the distribution is defined originally by $Q_{\text {cano }}\left(L^{(\alpha)}, L^{(\beta)}, L^{(\gamma)}\right)$, where $L^{(\alpha)}, L^{(\beta)}$, and $L^{(\gamma)}$ are respectively indices to specify the positions $\lambda^{(\alpha)}$, $\lambda^{(\beta)}$, and $\lambda^{(\gamma)}$ in the 3D-RC space. Then, we convert $\left[L_{i}^{(\alpha)}, L_{j}^{(\beta)}, L_{k}^{(\gamma)}\right]$ to: $\lambda_{l}^{(\alpha)}=0.5\left\{\left[\lambda_{i}^{(\alpha)}\right]_{\min }+\right.$ $\left.\left[\lambda_{i}^{(\alpha)}\right]_{m a s}\right\}$, where $i=1, \ldots, n_{v s}(\alpha), \lambda_{j}^{(\beta)}=0.5\left\{\left[\lambda_{j}^{(\beta)}\right]_{\min }+\left[\lambda_{j}^{(\beta)}\right]_{\text {mas }}\right\}$, where $j=1, \ldots, n_{v s}(\beta)$, and $\lambda_{j}^{(\beta)}=0.5\left\{\left[\lambda_{j}^{(\gamma)}\right]_{\min }+\left[\lambda_{j}^{(\gamma)}\right]_{\text {mas }}\right\}, k=1, \ldots, n_{v s}(\gamma)$. See Supplementary Table S3 for values of $\left[\lambda_{i}^{(h)}\right]_{\text {min }}$, $\left[\lambda_{i}^{(h)}\right]_{\max }$, and $n_{v s}(h)(h=\alpha, \beta, \gamma)$. Then, $Q_{\text {cano }}\left(\lambda^{(\alpha)}, \lambda^{(\beta)}, \lambda^{(\gamma)}\right)$ is normalized so that the highest density is set to 1 . Contour levels are presented by colors in inset.

\section{Distribution of ligands around $\mathrm{mSin} 3 \mathrm{~B}$.}


We computed the spatial density $\rho_{C G}^{(s)}(\boldsymbol{r})$ for the geometric center of the ligands (Figure $2)$. At the low-contour level $\left(\rho_{C G}^{(s)}(\boldsymbol{r})=0.005 \rho_{0}\right)$; green-colored contours), the ligands distributed almost everywhere around $\mathrm{mSin} 3 \mathrm{~B}$ for all systems. This result is natural indicating that the sampling was done widely. With increasing the density level, the ligands tended to be localized at some surface regions of $\operatorname{mSin} 3 \mathrm{~B}\left(\rho_{C G}^{(s)}(\boldsymbol{r})=0.05 \rho_{0}\right.$; blue-colored contours). At the high-density level $\left(\rho_{C G}^{(s)}(\boldsymbol{r})=0.5 \rho_{0}\right.$; red-colored contours), a high-density conformational cluster (labeled Cluster $\mathrm{A}$ in the figure) can be identified in the cleft of $\mathrm{mSin} 3 \mathrm{~B}$ for sertraline and $\mathrm{YN} 3$, whereas acitretin did not show a remarkable cluster in the cleft. This indicates that the ligand-mSin3B binding for sertraline and YN3 is stronger than that for acitretin.

Figure $2 \mathrm{~d}$ displays the REST/NRSF-mSin3B complex structure solved by the NMR experiment ${ }^{22}$ from the same orientation of Figure 1a-c. A view from a different orientation is presented in Supplementary Figure S13a. The binding cleft of mSin3B is hydrophobic (Supplementary Figure S13), and two residues Leu 46 and Leu 49 of the LIML sequence bind to the cleft forming a hydrophobic core with Val 75, Phe 93, and Phe 96 of mSin3B. See Introduction for the LIML sequence. Interestingly, only the geometrical center of sertraline overlaps the position of Leu 46 and Leu 49 of the LIML sequence (black broken-line circle) in the REST/NRSF-mSin3B complex, when the spatial density is viewed at the contour level of $\rho_{C G}^{(s)}(\boldsymbol{r})=0.5 \rho_{0}$. Therefore, sertraline forms a tighter hydrophobic core with the binding cleft of $\mathrm{mSin} 3 \mathrm{~B}$ than the other ligands do.

We also observed a high-density cluster (Cluster B in Figure 2) near the Cterminal of mSin3B. Because the C-terminal tail was restrained (see the Materials-andMethods and Supplementary Section 2), the C-terminal tail did not fluctuate largely in the simulation in spite that the C-terminal tail is exposed to solvent. Therefore, it is likely that Cluster B was induced by this less-fluctuating C-terminal tail: Cluster B is an artificial cluster.

Fig. 2 


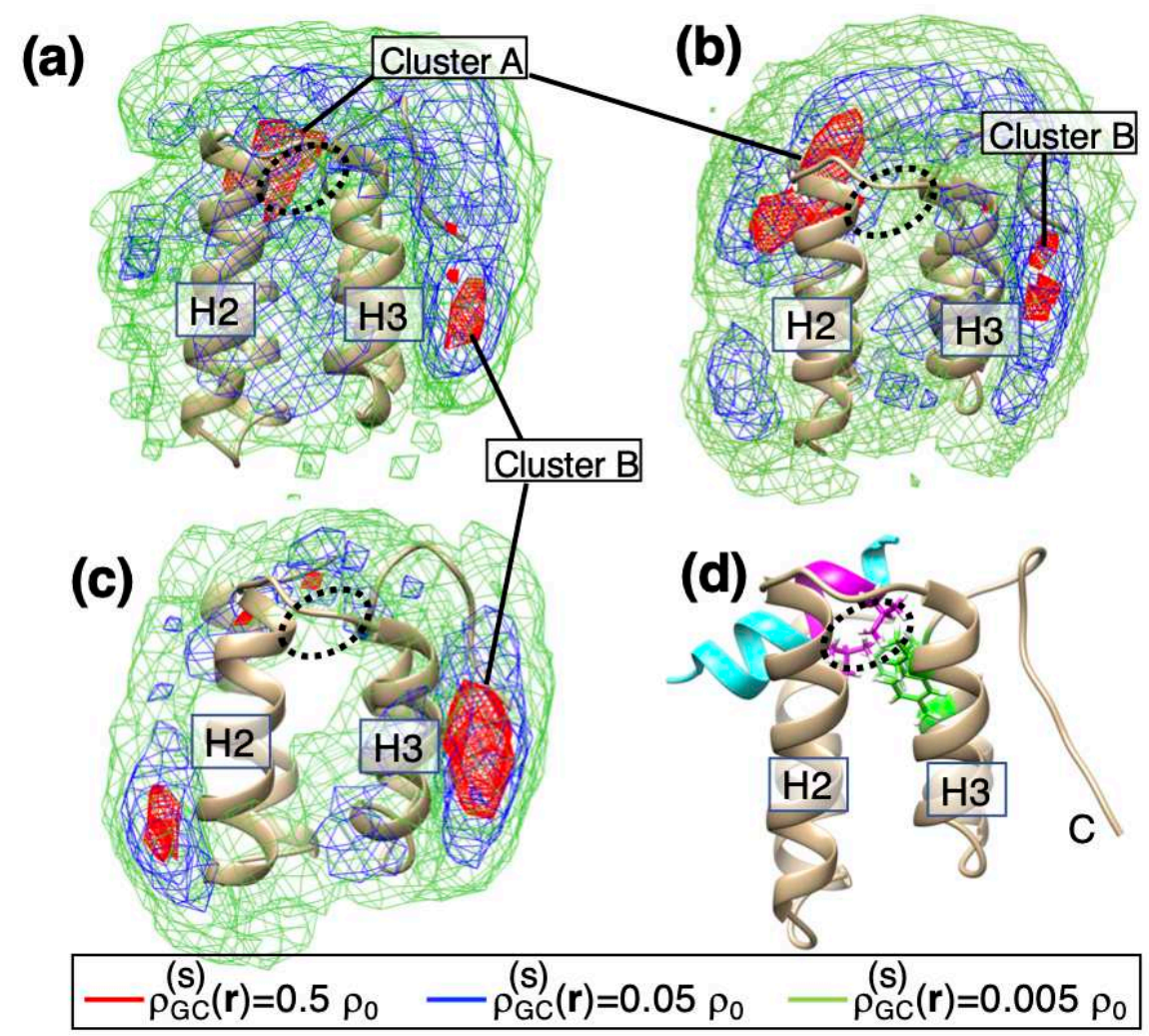

Figure 2. Spatial density $\rho_{C G}^{(s)}(\boldsymbol{r})$ of the geometric center (GC) at position $\boldsymbol{r}$ for (a) the sertraline-mSin3B, (b) YN3-mSin3B, and (c) acitretin-mSin3B systems in the 3D real space. See Supplementary Section 4 for procedure to calculate $\rho_{C G}^{(s)}(\boldsymbol{r})$. Contour levels are shown in inset where $\rho_{0}=0.001$. Displayed structure of $\mathrm{mSin} 3 \mathrm{~B}$ is that after NPT simulation for each system, where labels $\mathrm{H} 2$ and $\mathrm{H} 3$ represent helix 2 and helix 3 , respectively. The high-density cluster $\left(\rho_{C G}^{(s)}(\boldsymbol{r})>0.5 \rho_{0}\right)$ in the cleft of mSin3B (PAH1) is named as Cluster A, and one near the N-terminal of mSin3B as Cluster B. (d) NMR structure of REST/NRSFmSin3B complex (PDB ID: 2CZY), where cyan-colored model is REST/NRSF, and the magenta-colored segment is the LIML sequence of REST/NRSF. Label C indicates the position of the C-terminal tail of mSin3B. Two magenta-colored side-chains are Leu 46 and Leu 49 of the LIML sequence. Black brokenline circle indicates the position of the two sidechains. Those circles in panels (a), (b), and (c) are presented to indicate the sidechain position of Leu 46 and Leu 49. Green-colored sidechains are Val 75, Phe 93, and Phe 96 of mSin3B (see also green-colored sidechains of Supplementary Figure S12a), which form a hydrophobic core with Leu 46 and Leu 49 of the LIML sequence.

\section{Radial distribution function of ligand-cleft distance.}

Figure 2 was insightful to guess the ligand-mSin3B interaction. Nevertheless, $\rho_{C G}^{(s)}(\boldsymbol{r})$ was calculated using the geometrical centers of the ligands. There is a possibility that some parts of the ligands contacted to the hydrophobic cleft tightly even when the 
geometrical center was distant from the cleft. For instance, acitretin is a long molecule, and then the head or tail of acitretin (Supplementary Figure S4) may be inserted to the cleft keeping the geometrical center out of the cleft.

To make clear this point, we calculated a radial distribution function (RDF) between the ligand and three residues Val 75, Phe 93, and Phe 96 located in the mSin3B cleft. The computation procedure for RDF is given in Supplementary Section 6. First, we define the minimum heavy-atomic distance between the ligand and one of the three residues: $r_{R}^{(s)}$ where the subscript $R$ is the residue specifier $(R=$ Val 75, Phe 93, or Phe 96) and the superscript $s$ is the system specifier defined before. Then, the RDF $p\left(r_{R}^{(s)}\right)$ was calculated for each of the three distances.

Figure 3 demonstrates the resultant RDFs. For all of the systems, the function $p\left(r_{P h e 96}^{(s)}\right)$ exhibited a peak at $3.5 \AA$ A regardless of the peak height. This result is plausible because the residue Phe 96 is located at the entrance of the cleft (Supplementary Figure S13): The ligand can contact to Phe 96 without sinking into the cleft. More important RDFs are $p\left(r_{\text {Val75 }}^{(s)}\right)$ and $p\left(r_{P h e 93}^{(s)}\right)$ because Val 75 and Phe 93 are located at the bottom of the cleft. Apparently, the peaks of the RDFs at $r_{R}^{(s)} \sim 4 \AA$ for the acitretin-mSin3B system were considerably smaller than those for sertraline-mSin3B and YN3-mSin3B systems. This means that acitretin did not interact frequently or tightly with the bottom of the cleft. The highest peaks were from the sertraline-mSin3B system (Figure 3a), and the peaks from the $\mathrm{YN} 3-\mathrm{mSin} 3 \mathrm{~B}$ were intermediate between sertraline and acitretin (Figure $3 \mathrm{~b}$ ). These results suggest that sertraline may resemble the ligand-receptor interaction mode found in the REST/NRSF-mSin3B complex.

Fig. 3

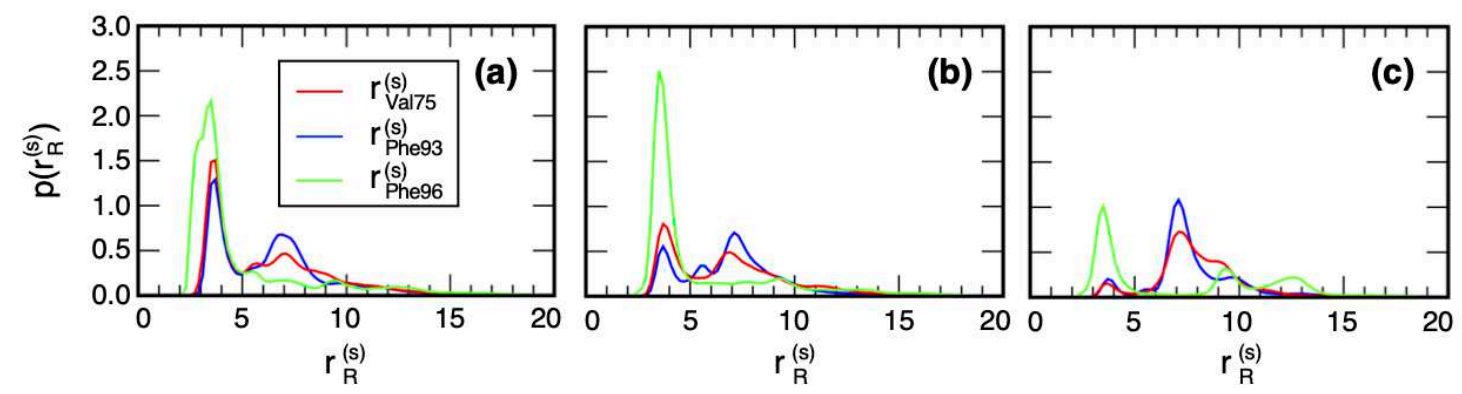

Figure 3. Radial distribution functions (RDFs) $p\left(r_{R}^{(s)}\right.$ ) for (a) the sertraline-mSin3B, (b) YN3-mSin3B, and (c) acitretin-mSin3B systems. Three RDFs $p\left(r_{V a l 75}^{(s)}\right), p\left(r_{P h e 93}^{(s)}\right)$, and $p\left(r_{P h e 96}^{(s)}\right)$ are shown by different colors as indicated in inset of panel (a). See Supplementary Section 6 for procedure to calculate RDF. 
To investigate more the ligand-receptor interactions for the sertraline-mSin3B system, we calculated the spatial density $\rho_{G C A}^{(s)}(\boldsymbol{r})$ of the geometrical center of Ring A of sertraline: See Supplementary Figure S1b for the position of Ring A and Supplementary Eq. S4 for the procedure to calculate $\rho_{G C A}^{(s)}(\boldsymbol{r})$. Figure 4 illustrates $\rho_{G C A}^{(s)}(\boldsymbol{r})$ and $\rho_{G C}^{(s)}(\boldsymbol{r})$ with the contour level of $0.5 \rho_{0}$. Apparently, Ring A occupied the deeper position of the cleft than the geometrical center of the entire sertraline did. Thus, Ring A was closer to Val 75, Phe 93, and Phe 96 of mSin3B.

Fig. 4

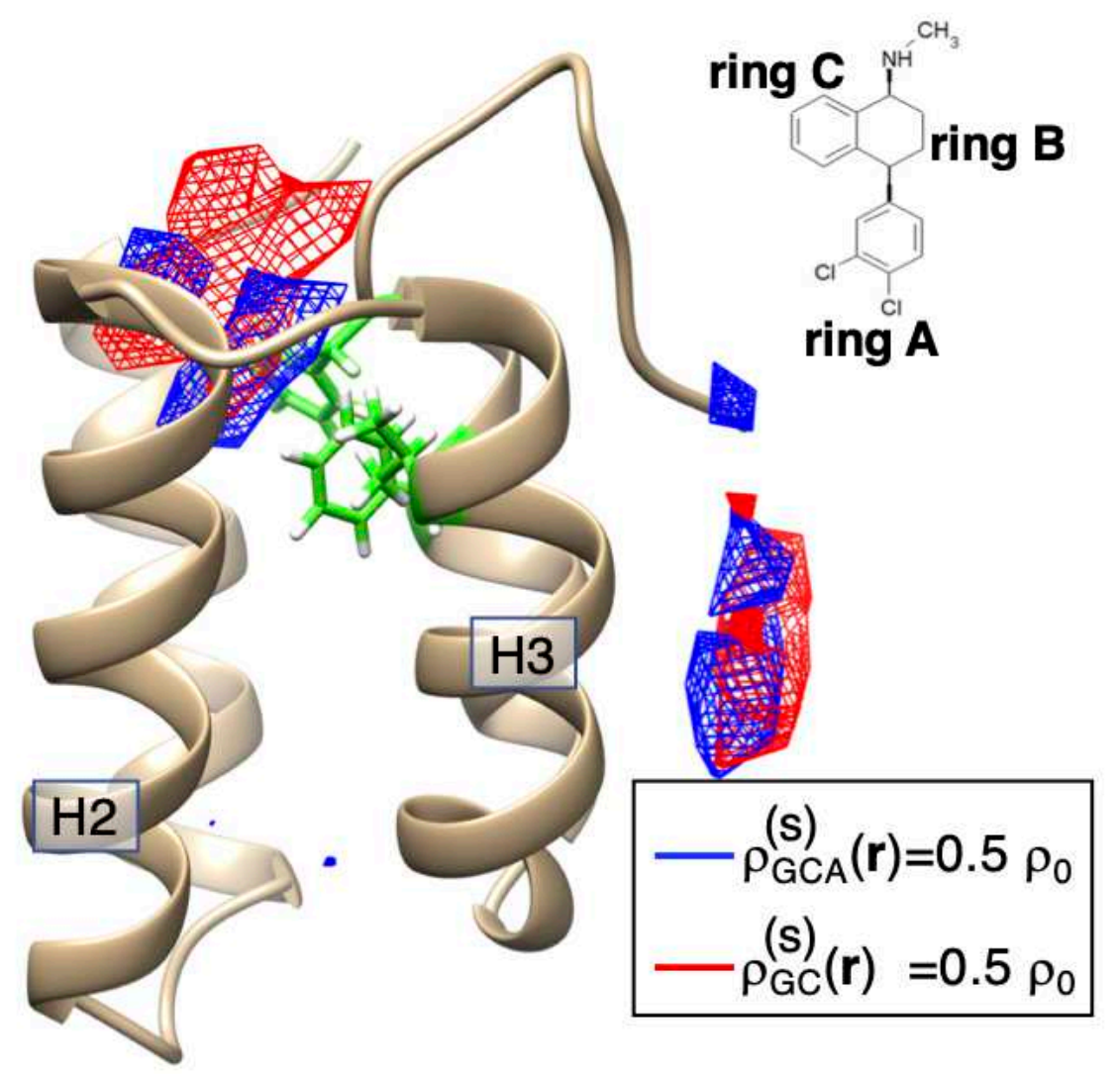

Figure 4. Spatial density $\rho_{C G A}^{(s)}(\boldsymbol{r})$ (blue-colored contours) for the geometric center of Ring A of sertraline in the sertraline-mSin3B system, where the contour level is $\rho_{C G A}^{(s)}(\boldsymbol{r})=0.5 \rho_{0}\left(\rho_{0}=0.001\right)$. Chemical structure of sertraline is also shown. Red-colored contours are $\rho_{C G}^{(s)}(\boldsymbol{r})$ for the geometric center of the entire sertraline. Labels $\mathrm{H} 2$ and $\mathrm{H} 3$ represent helices 2 and 3, respectively. Green-colored residues are Val 75, Phe 93, and Phe 96 of mSin3B. See Supplementary Section 4 for procedure to calculate $\rho_{C G A}^{(s)}(\boldsymbol{r})$.

Orientation of ligands bound to $\mathrm{mSin} 3 \mathrm{~B}$. 
We checked the sertraline-mSin3B complex structures detected in the cleft of mSin3B with the high-density region, $\rho_{C G A}^{(s)}(\boldsymbol{r})>0.5 \rho_{0}$, of Ring A. Figure 5 demonstrates some conformations picked randomly from the high-density region. This figure also displays Val 75, Phe 93, and Phe 96 of mSin3B. Here we judged that Ring A was contacting to the residues when $r_{R}^{(s)}<4.0 \AA$ was satisfied. In Figure 5a, Ring A contacted to Phe 93 and Phe 96, whereas that in Figure 5b did not to any of the three residues. In Figure 5c Ring A contacted to all of the three residues in Figure 5c and to Val 75 in Figure 5d. Examining a number of snapshots, we presumed that ligand-receptor contacts shown in Figure $3 \mathrm{a}$ were contributed mainly by Ring A.

Fig. 5

(a)

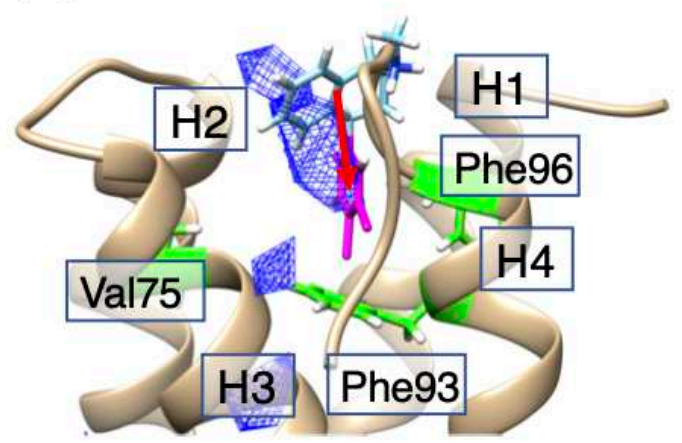

(c)

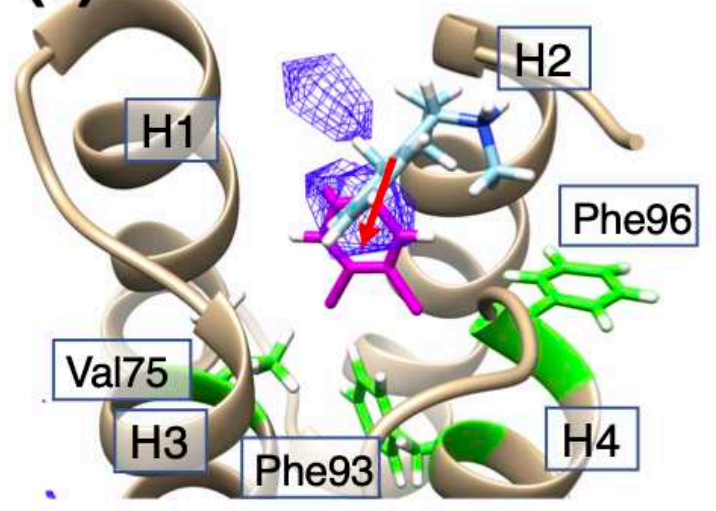

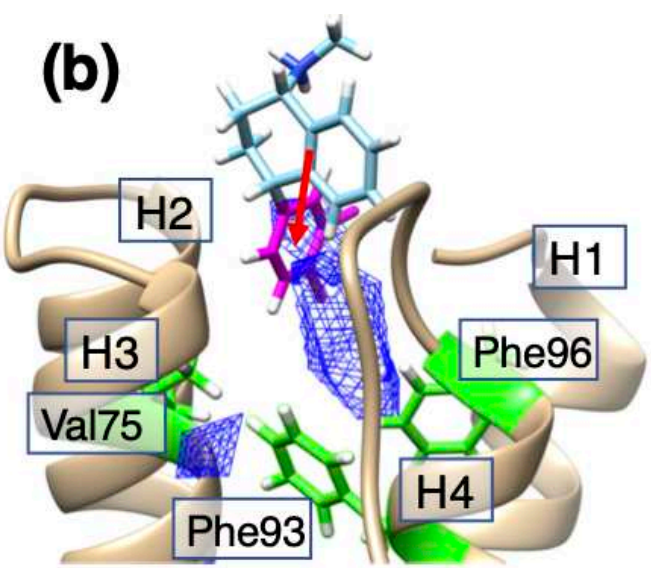

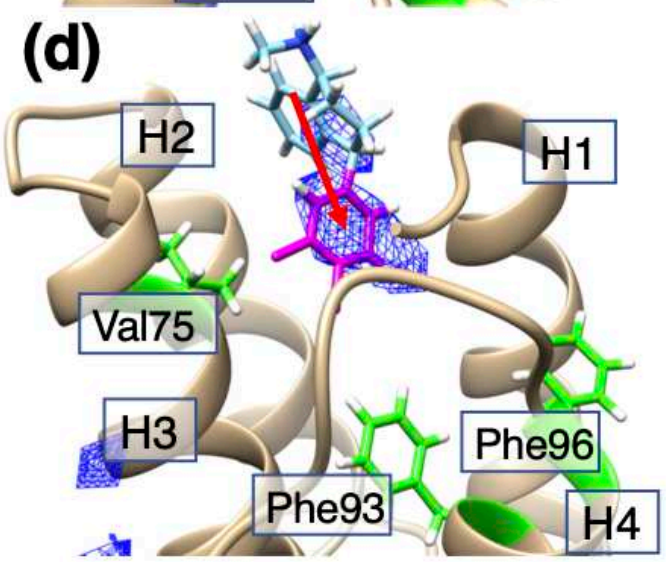

Figure 5. (a)-(d) Sertraline-mSin3B complexes picked randomly from regions of $\rho_{C G A}^{(s)}(\boldsymbol{r})>0.5 \rho_{0} \quad\left(\rho_{0}=\right.$ 0.001), which are shown by blue-colored contours. Magenta-colored portion is Ring A of Sertraline. Redcolored vectors are those pointing from Ring-BC geometrical center to that of Ring A (see Supplementary Figure S1b for positions of Ring A and BC). Green-colored residues are Val 75, Phe 93, and Phe 96 of mSin3B. Labels H1,.., H4 are helices $1-4$ of mSin3B (PAH1 domain). 
Fig. 5

Red-colored vectors in Figure 5 indicate the molecular orientations of the shown snapshots. See Supplementary Figure S2b for the definition of the sertraline's molecular orientation. Figure 5 indicates that Ring A was inserted into the binding cleft of $\mathrm{mSin} 3 \mathrm{~B}$ and that Ring BC was left behind. Here, to investigate statistically the sertraline's orientation, we introduced a unit vector $\boldsymbol{e}_{i}^{(s)}$ parallel to the vector from ring BC to Ring A for snapshot $i$, and calculated average, $\left\langle\boldsymbol{e}^{(s)}(\boldsymbol{r})\right\rangle$, of the unit vectors in each cube centered at $\boldsymbol{r}$. The detailed procedure for averaging is presented in Supplementary Section 7. Figure 6 demonstrates the spatial pattern of $\left\langle\boldsymbol{e}^{(s)}(\boldsymbol{r})\right\rangle$ for the sertraline$\mathrm{mSin} 3 \mathrm{~B}$ system viewed from two different directions. This figure also indicates that Ring A tends to be inserted in the cleft of $\mathrm{mSin} 3 \mathrm{~B}$, which is consistent to Figure 5.

Fig. 6
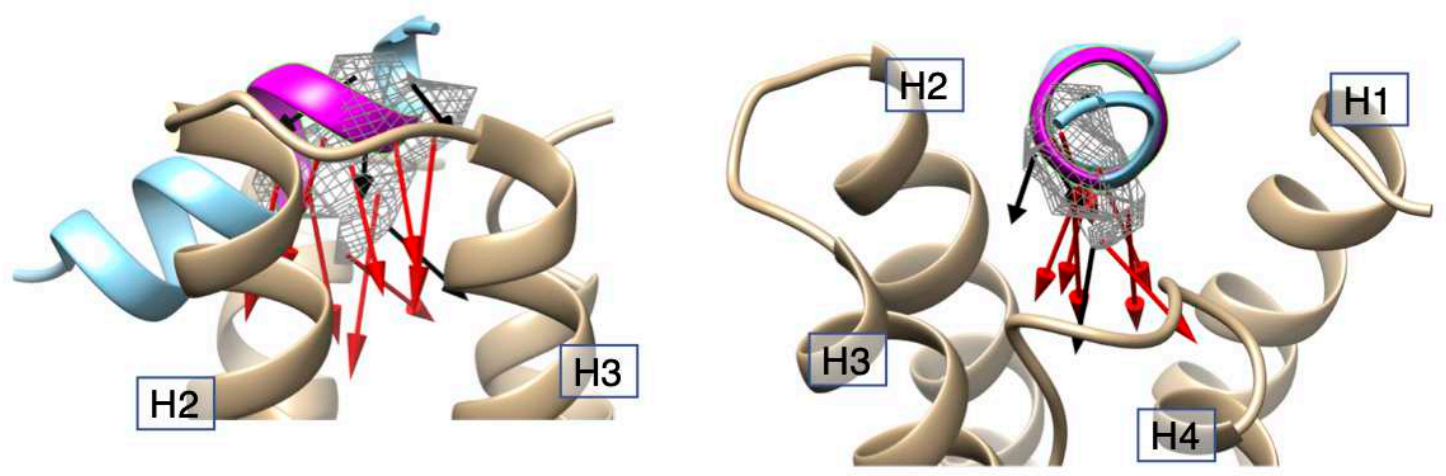

Figure 6. Spatial pattern of $\left\langle\boldsymbol{e}^{(s)}(\boldsymbol{r})>\right.$ for sertraline in the cleft of mSin3B viewed from two different directions. Red and black vectors are those with $\left|<\boldsymbol{e}^{(s)}(\boldsymbol{r})>\right| \geq 0.5$ and $\left|<\boldsymbol{e}^{(s)}(\boldsymbol{r})>\right|<0.5$, respectively. Gray contours represent the high-density regions of $\rho_{C G}^{(s)}(\boldsymbol{r})>0.5 \rho_{0}\left(\rho_{0}=0.001\right)$. The vectors $\left\langle\boldsymbol{e}^{(s)}(\boldsymbol{r})>\right.$ are shown in regions with $\rho_{C G}^{(s)}(\boldsymbol{r})>0.5 \rho_{0}$. This figure also displays REST/NRSF bound to mSin $3 \mathrm{~B}$, and the magenta-colored segment is the LIML sequence of REST/NRSF. Note that REST/NRSF is not involved in the current simulation. The shown structure is the REST/NRSF-mSin3B complex structure (PDB ID: 2CZY). Labels H1,.., H4 are helices 1,.., 4 of mSin3B (PAH1 domain).

We also calculated $\left\langle\boldsymbol{e}^{(Y N 3)}(\boldsymbol{r})\right\rangle$ for the YN3-mSin3B (Figure 7) and acitretin-mSin3B (Figure 8) systems. See Supplementary Figures S3b and S4b for the definition of the molecular orientations for YN3 and acitretin. Figure 8 shows that the 
acitretin's orientation tends to be parallel or anti-parallel to the helical cylinder of REST/NRSF in the REST/NRSF-mSin3B complex. We presume that these acitretin's orientations have an advantage to fit the whole acitretin's framework to the binding cleft of $\mathrm{mSin} 3 \mathrm{~B}$. To stabilize one of the parallel or anti-parallel orientations of acitretin, an additional inter-molecular interaction is required, which works differently between the two orientations. We consider that there is no such interaction to stabilize effectively one of the two orientations for the acitretin-mSin3B system.

Fig. 7
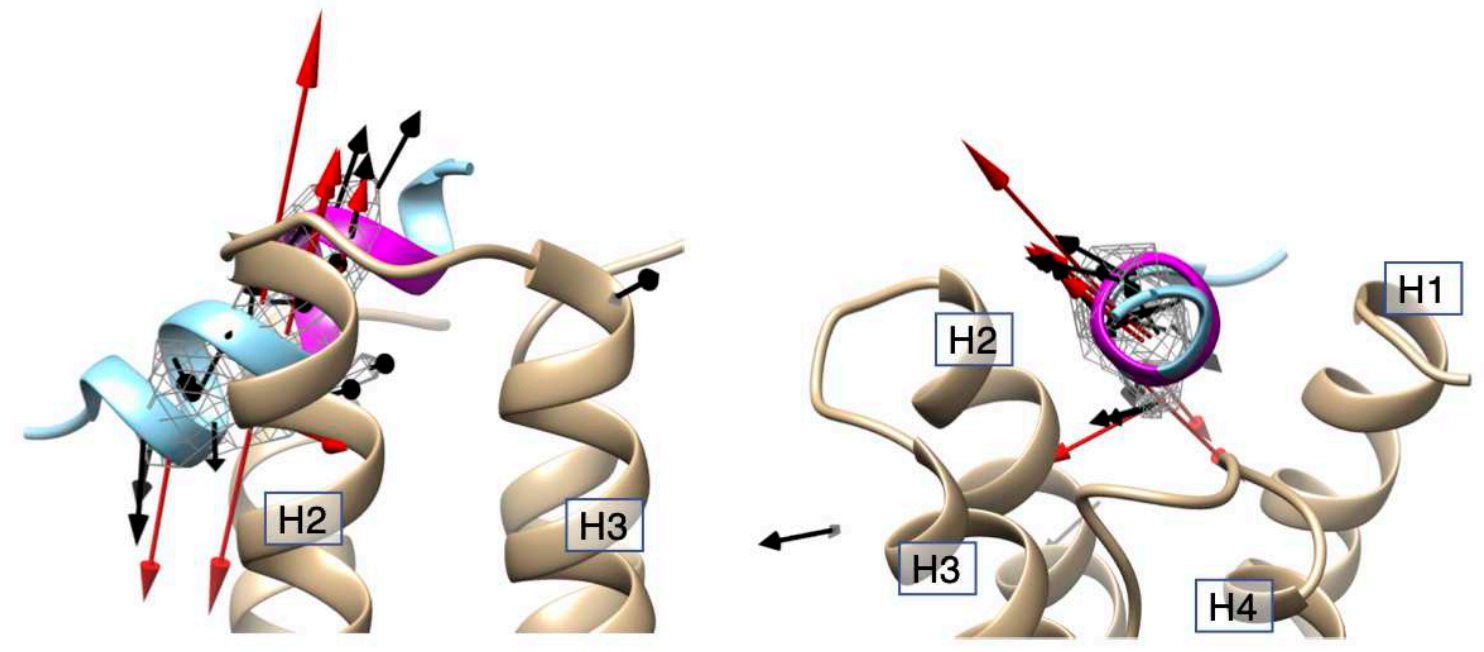

Figure 7. Spatial pattern of $\left\langle\boldsymbol{e}^{(s)}(\boldsymbol{r})>\right.$ for YN3 in the cleft of mSin3B viewed from two different directions. Gray contours represent the high-density regions of $\rho_{C G}^{(s)}(\boldsymbol{r})>0.5 \rho_{0}\left(\rho_{0}=0.001\right)$. See caption of Figure 6 for more information.

Fig. 8 

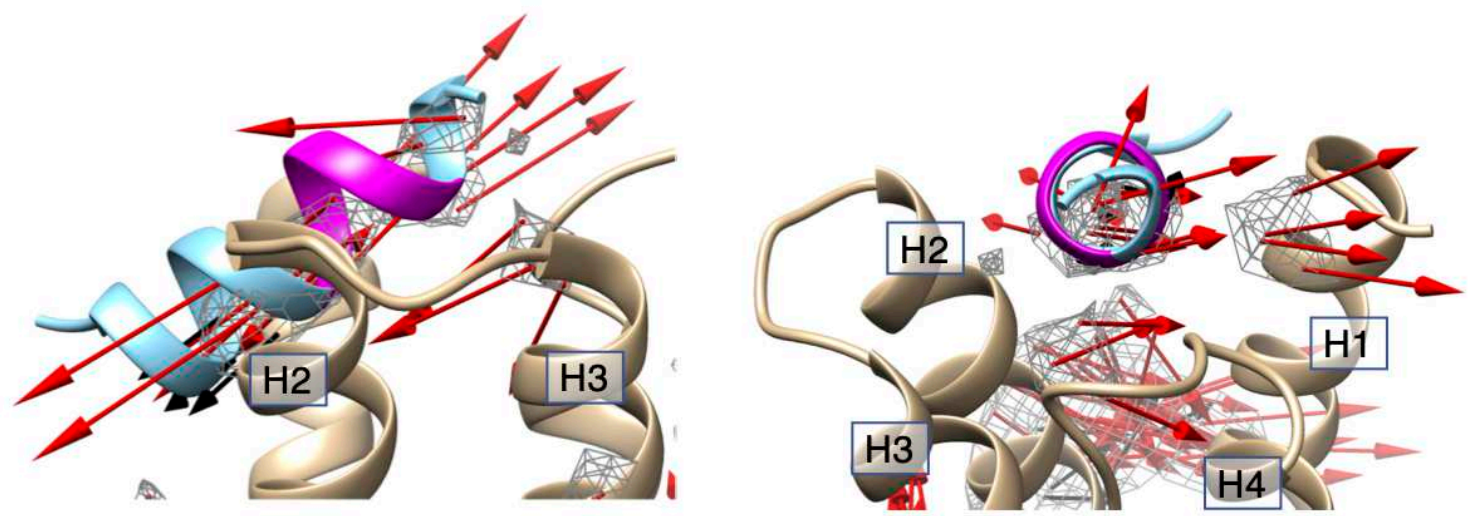

Figure 8. Spatial pattern of $\left\langle\boldsymbol{e}^{(s)}(\boldsymbol{r})>\right.$ for acitretin in the cleft of mSin3B viewed from two different directions. Gray contours represent the high-density regions of $\rho_{C G}^{(s)}(\boldsymbol{r})>0.1 \rho_{0}\left(\rho_{0}=0.001\right)$. See caption of Figure 6 for more information.

On the other hand, the YN3's orientations are not aligned to the helical cylinder of REST/NRSF. We presume that this tilting of YN3 to the helical cylinder is because YN3 is smaller than acitretin: YN3 may be responding to undulations of mSin3B's molecular surface in the cleft. This point is discussed later again.

\section{Flexibility of ligands' framework}

Figure 5 demonstrates the structural variety of the sertraline's framework. To quantify the flexibility of the ligand's framework, we calculated the distance distribution function, DDF, for a distance between two atoms set in each ligand. The procedure to calculate DDF for a distance is given in Supplementary Section 6.

Here, we picked the cyan- and orange-colored atoms in Figure 9a from the framework of each ligand, and calculated the distance between the two atoms: $r_{\text {atom-pair }}^{(s)}$.

The DDFs $P\left(r_{\text {atom-pair }}^{(s)}\right)$ for these distances are displayed in Figure $9 \mathrm{~b}$. The average of the distance $<r_{\text {atom-pair }}^{(s)}>$ and its standard deviation (amount of fluctuations) $S D\left(r_{\text {atom-pair }}^{(s)}\right)$ were: $7.12 \AA$ and $0.37 \AA$ for sertraline, $6.01 \AA$ and $0.14 \AA$ for $\mathrm{YN} 3$, and 13.15 and $0.47 \AA$ for acitretin. The largest SD was assigned to acitretin. However, this does not mean that acitretin is the most flexible, because a long molecule may have generally a large SD even if the ligand is stiff, and because acitretin is the longest ligand of the three. We consider that the amount of fluctuations per unit length, $s d^{(s)}=$ 
$S D\left(r_{\text {atom-pair }}^{(s)}\right) /<r_{\text {atom-pair }}^{(s)}>$, is a better quantity to quantify the flexibility of the molecular flexibility. The resultant $s d^{(s)}$ was $5.20 \times 10^{-2}$ for sertraline, $2.33 \times$ $10^{-2}$ for $\mathrm{YN} 3$, and $3.57 \times 10^{-2}$ for acitretin. From these values, the framework of sertraline is the most flexible, acitretin has a considerably stiffer framework than sertraline does, and YN3 is stiffer than acitretin. The flexibility of the framework may be related to the ligand's molecular orientation as discussed later.

Fig. 9

(a)

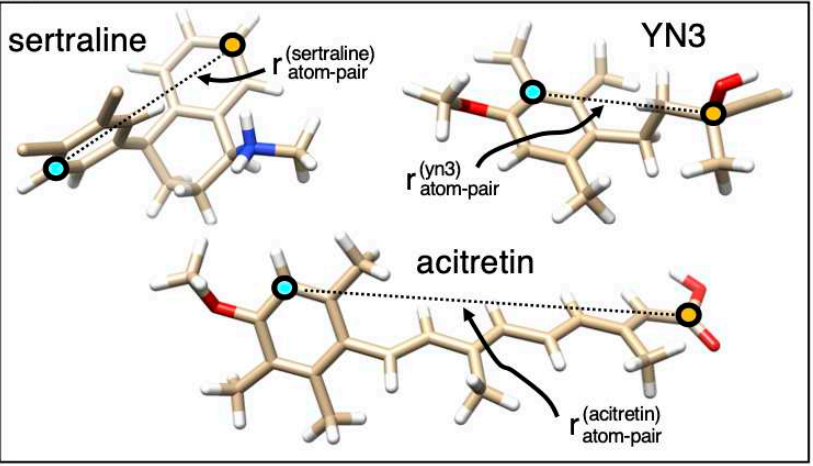

(b)

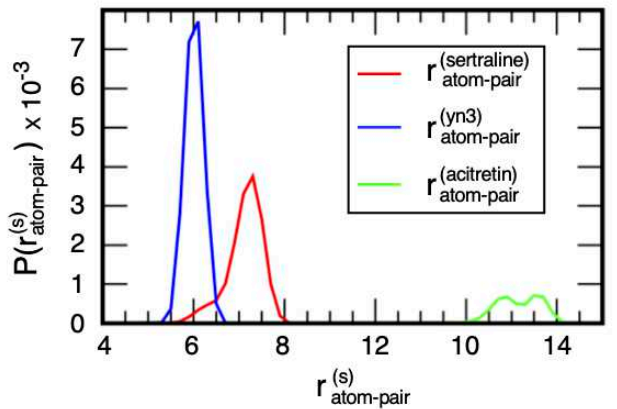

Figure 9. (a) Structures of the three compounds sertraline, YN3, and acitretin. For each compound, interatomic distance $r_{\text {atom-pair }}^{(s)}(s=$ sertraline, YN3, or acitretin) is defined between cyan- to orange-colored atoms. A cyan-colored atom, which is involved in a ring for each ligand, is not set on the ring rotation axis to detect the ring-rotational motions. (b) Distance distribution functions (DDFs) $P\left(r_{\text {atom-pair }}^{(s)}\right.$ for the three distances. See Supplementary Section 6 for procedure to calculate DDF.

\section{Discussion}

Starting from the initial conformations in that the ligands were distant from the cleft of mSin3B (Supplementary Figure S7-S9), only sertraline reproduced a similar intermolecular hydrophobic core as observed in the REST/NRSF-mSin3B complex (PDB ID: 2CZY): Ring A of sertraline, which is hydrophobic, bound deeply to the cleft of $\mathrm{mSin} 3 \mathrm{~B}$ with contacting to the hydrophobic sidechains sited in the cleft (Figure 5). Thus, the sertraline-mSin3B complex formation competes with the REST/NRSFmSin3B complex formation.

Now, we discuss the interactions of acitretin and YN3 with mSin3B. Acitretin has a low spatial density in the mSin3B's cleft (Figure 2c), which weakens the inhibitory activity partly. Furthermore, the framework of acitretin tends to be parallel or anti-parallel 
to the mSin3B's cleft (Figures 8 and 10c). Remember that acitretin can be regarded as a long and stiff rod as shown in the above section. The parallel or anti-parallel molecular orientation is advantageous to be fit to the cleft. If acitretin has a flexible framework, acitretin may insert a portion into the hydrophobic cleft by bending the framework.

Although YN3 had a high spatial density around the mSin3B's cleft (Figure 2c), YN3 did not sink deeply in the cleft and the hydrophobic core was not formed. This result is natural because YN3 can be regarded as a stiff rod. As discussed for acitretin above, the stiff rod fits well to the cleft taking the parallel or anti-parallel orientation. On the other hand, we presume that the slight tilt of $\left\langle\boldsymbol{e}^{(Y N 3)}(\boldsymbol{r})\right\rangle$ to the helix cylinder of NRSF/REST in the NRSF/RESTmSin3B complex is resulted from the small size of YN3 (Figure 9b). I.e., YN3 is can adopt to the jaggedness of the inside of the cleft. If YN3 has more flexibility, this compound may exert the inhibitory activity by inserting a molecular portion into the cleft with varying the molecular conformation. On the other hand, the added flexibility may induce binding of YN3 to the other surfaces of mSin3B than the cleft. These competitive effects of the molecular flexibility cannot be assessed only from the chemical structure of the compound.

We also note another chemical property of YN3 and acitretin, which is disadvantageous for interacting with hydrophobic cleft of mSin3B: Both rings in these compounds have an oxygen atom (Supplementary Figures S3 and S4), which can interact to hydrophilic residues in mSin3B electrostatically or by hydrogen bonding.

Therefore, we note that the current simulation is useful to judge the probable complex structure. The current simulation provides the thermally equilibrated conformational ensemble of the molecular system, where the atomic interactions, the structural flexibility, and the solvent effect are explicitly considered.

The conventional structure-activity relationship (SAR) study has been based on the chemical structures and target-binding activity data. Figure 10a shows one of the possible alignments of the three compounds based on their chemical structures. Each compound has an aromatic ring corresponding to Ring $\mathrm{A}$ and each aromatic ring has side chains in the para position. While sertraline has $\mathrm{Cl}$ atoms on Ring $\mathrm{A}, \mathrm{YN} 3$ and acitretin have methoxy groups whose volumes are close to that of the $\mathrm{Cl}$ atom. Instead of Ring $\mathrm{C}$ of sertraline, YN3 and acitretin have triple and double bonds, respectively. These bonds are $\pi$-electron rich, and can form $\mathrm{CH}-\pi$ interaction as same as aromatic rings. However, such SAR analysis could not explain presence and absence of the medulloblastoma cellgrowth inhibition activities of their compounds (Figure 10b). The present MD simulation study predicted the molecular orientations of the compounds (Figure 10c) and the presence/absence of the hydrophobic core in the cleft of mSin3B (Figure 2). Importantly, 
only sertraline could reproduce the binding mode in the complex of NRSF/REST and mSin3B. We emphasize that the current simulation method, the GA-guided mD-vcMD simulation, produces a thermodynamically acceptable ensemble consisting of various conformations (bound and unbound conformations), and importantly a thermodynamic weight is assigned to each snapshot in the ensemble.

Fig. 10

Sertraline

(a)
YN3

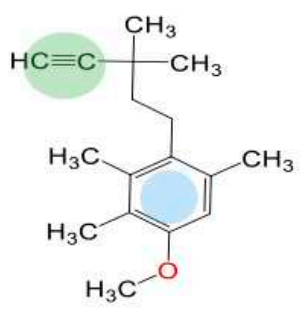

\section{Acitretin}

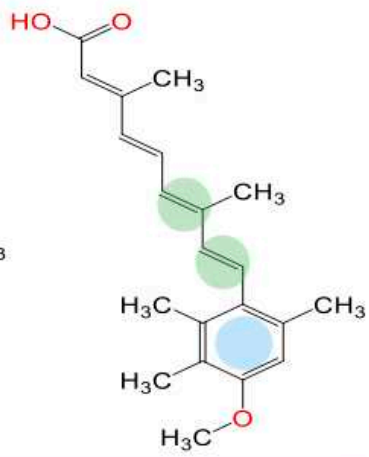

(b)

Medulloblastoma cell growth inhibition

Active Inactive Inactive
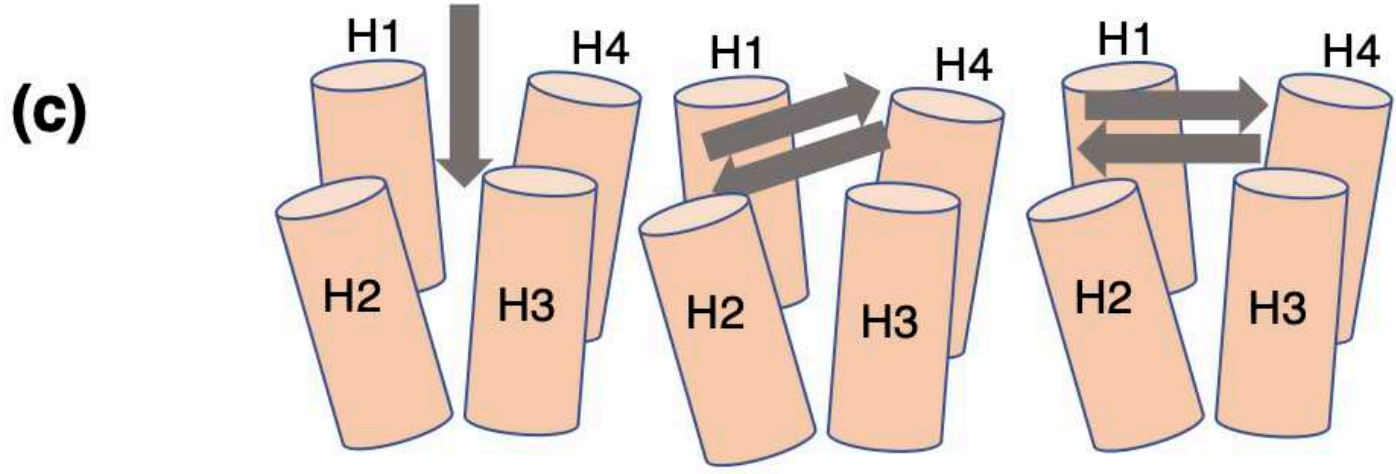

Figure 10. (a) Chemical structures of $\mathrm{mSin} 3 \mathrm{~B}$ binders, and their orientation aligned from head to tail sides. Rings $\mathrm{A}, \mathrm{B}$ and $\mathrm{C}$ of sertraline are depicted in magenta, black, and orange, respectively. Blue circles represent aromatic rings that are expected to interact with the hydrophobic cleft of $\mathrm{mSin} 3 \mathrm{~B}$ from conventional structure-activity relationship (SAR). Green circles represent $\pi$-electron rich regions. (b) Presence/absence of Medulloblastoma cell-growth inhibition activities for the compounds. (c) The actual orientations of the compounds with respect to the cleft of $\mathrm{mSin} 3 \mathrm{~B}$, predicted by the present MD simulation study. 
The preceding study ${ }^{31}$ classified 52 compounds into two pharmacophores, A and B, based on their chemical structures (see Fig. 2 of Ref. 31), where sertraline belongs to Pharmacophore A and the YN3 to Pharmacophore B. Because acitretin has a structural similarity with YN3 apparently, acitretin belongs to Pharmacophore B. Based on Figures 6-8 and Figure 10c, the compounds belonging to Pharmacophore A have the parallel or anti-parallel orientation, and the compound belonging to Pharmacophore $\mathrm{B}$ has a perpendicular orientation. Therefore, the current MD procedure is useful if it is used with the pharmacophore analysis.

\section{Conclusions}

Binding of the compounds to mSin3B (PAH1 domain) was investigated by the GAguided $\mathrm{mD}-\mathrm{VcMD}$ simulation. This method produced useful quantities such as the spatial density of the ligand around the receptor (Figure 2), the intermolecular contact patterns (Figure 3), the propensity of molecular orientation (Figures 5-8), and the ligand flexibility (Figure 9). From these analyses, we showed that only sertraline produces a similar intermolecular binding mode observed in the REST/NRSF-mSin3B complex. Figure 11 is a schematic drawing to design an inhibitor. Given a framework of the compound, by adding a hydrophobic sidechain to the framework, the hydrophobic core is formed between the sidechain and the hydrophobic cleft of $\mathrm{mSin} 3 \mathrm{~B}$. The flexibility of the compound's framework may increase the binding affinity, although a long and stiff framework may decrease the binding affinity. In general, it is difficult to specify the effect of the modification to the biological activity (i.e., inhibitory activity in the present study) only from the compound's chemical structure. In contrast, the GA-guided mD-VcMD is useful to identify the effect with analyzing the thermally equilibrated conformational ensemble.

Fig. 11 


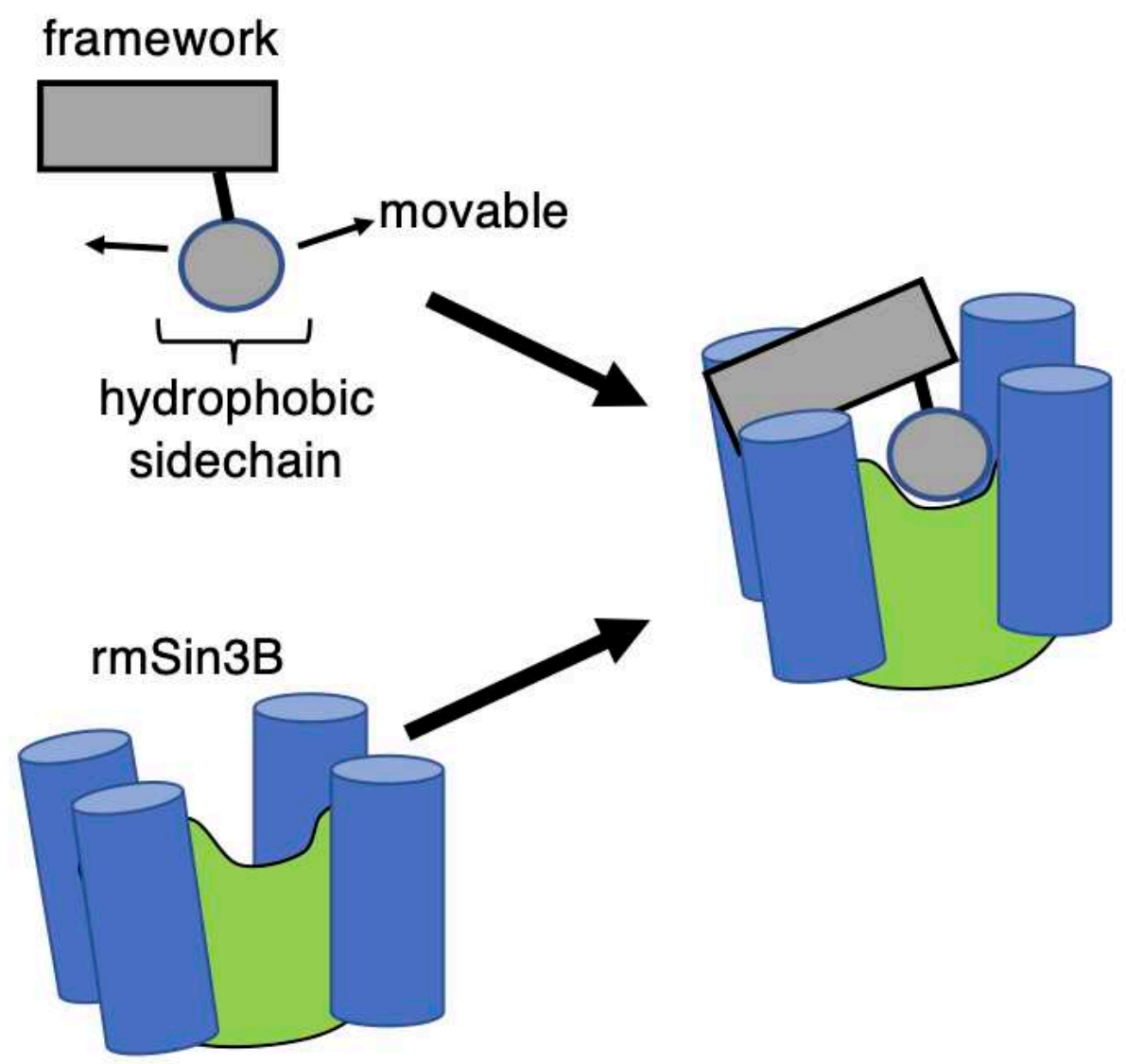

Figure 11. Scheme of a compound that binds to the cleft of $\mathrm{mSin} 3 \mathrm{~B}$.

Because REST/NRSF is an intrinsically disordered segment that can bind to multiple proteins ${ }^{3,4}$ as mentioned in Introduction, the current study is an example to design a compound that can inhibit binding of an intrinsically disorder segment to a protein receptor.

The 3D models have been submitted to the Biological Structure Model Archive (BSM-Arc) of the PDBj under BSM-ID BSM00020 (https://bsma.pdbj.org/entry/20), which are freely available ${ }^{53}$.

\section{Acknowledgements}

J. H. was supported by JSPS KAKENHI Grant No. 16K05517 and by the Development of Core Technologies for Innovative Drug Development Based Upon IT from Japan Agency for Medical Research and Development (AMED). N. K. was supported by a JSPS KAKENHI Grant (No. JP20H03229). K. K. was supported by JSPS KAKENHI Grant No. 16K18526. This work was supported as an HPCI System Research Project (Project IDs: hp190017, hp190018, hp190027, hp200063, hp200090, and hp200025). Simulations 
were performed on the TSUBAME3.0 supercomputers at the Tokyo Institute of Technology, in part under the Cooperative Research Program of the Institute for Protein Research, Osaka University, CR-19-05 and CR-20-05.

\section{Author contributions}

All authors contributed equally.

\section{References}

1. Schoenherr, C. J. \& Anderson, D. J. The neuron-restrictive silencer factor (NRSF): A coordinate repressor of multiple neuron-specific genes. Science 267, 1360-1363 (1995).

2. Chong, J. A., Tapia-Ramirez, J., Kim, S., Toledo-Aral, J. J., Zheng, Y., Boutros, M. C., Altshuller, Y. M., Frohman, M. A., Kraner, S. D. \& Mandel, G. REST: A mammalian silencer protein that restricts sodium channel gene expression to neurons. Cell 80, 949-957 (1995).

3. Bruce, A. W., Donaldson, I. J., Wood, I. C., Yerbury, S. A., Sadowski, M. I., Chapman, M., Göttgens, B. \& Buckley, N. J. Genome-wide analysis of repressor element 1 silencing transcription factor/neuron-restrictive silencing factor (REST/NRSF) target genes. Proc. Natl. Acad. Sci. USA 101, 10458-10463 (2004).

4. Rockowitz, S. \& Zheng, D. Significant expansion of the REST/NRSF cistrome in human versus mouse embryonic stem cells: Potential implications for neural development. Nucleic Acids Res. 43, 5730-5743 (2015).

5. Lawinger, P., Venugopal, R., Guo, Z. S., Immaneni, A., Sengupta, D., Lu, W., Rastelli, L., Carneiro, A. M. D., Levin, V., Fuller, G. N., Echelard, Y. \& Majumder, S. The neuronal repressor REST/NRSF is an essential regulator in medulloblastoma cells. Nat. Med. 6, 826-831 (2000).

6. Fuller, G. N., Su, X., Price, R. E., Cohen, Z. R., Lang, F. F., Sawaya, R. \& Majumder, S. Many human medulloblastoma tumors overexpress repressor element-1 silencing transcription (REST)/neuronrestrictive silencer factor, which can be functionally countered by REST-VP16. Mol. Cancer Ther. 4, 343-349 (2005).

7. Dhall, G. Medulloblastoma. J. Child Neurol. 24, 1418-1430 (2009).

8. Conti, L., Crisafulli, L., Caldera, V., Tortoreto, M., Brilli, E., Conforti, P., Zunino, F., Magrassi, L., Schiffer, D. \& Cattaneo, E. REST controls self-renewal and tumorigenic competence of human glioblastoma cells. Plos One 7, e38486 (2012).

9. Kamal, M. M., Sathyan, P., Singh, S. K., Zinn, P. O., Marisetty, A. L., Liang, S., Gumin, J., El-Mesallamy, H. O., Suki, D., Colman, H., Fuller, G. N., Lang, F. F. \& 
Majumder, S. REST regulates oncogenic properties of glioblastoma stem cells. Stem Cells 30, 405-414 (2012).

10. Zuccato, C., Tartari, M., Crotti, A., Goffredo, D., Valenza, M., Conti, L., Cataudella, T., Leavitt, B. R., Hayden, M. R., Timmusk, T., Rigamonti, D. \& Cattaneo, E. Huntingtin interacts with REST/NRSF to modulate the transcription of NRSEcontrolled neuronal genes. Nat. Genet. 35, 76-83 (2003).

11. Zuccato, C. \& Cattaneo, E. Role of brain-derived neurotrophic factor in Huntington's disease. Prog. Neurobiol. 81, 294-330 (2007).

12. Bithell, A., Johnson, R. \& Buckley, N. J. Transcriptional dysregulation of coding and non-coding genes in gellular models of Huntington's disease. Biochem. Soc. Trans. 37, 1270-1275 (2009).

13. Buckley, N. J., Johnson, R., Zuccato, C., Bithell, A. \& Cattaneo, E. The role of REST in transcriptional and epigenetic dysregulation in Huntington's disease. Neurobiol. Dis. 39, 28-39 (2010).

14. Uchida, H., Ma, L. \& Ueda, H. Epigenetic gene silencing underlies C-fiber dysfunctions in neuropathic pain. J. Neurosci. 30, 4806-4814 (2010).

15. Willis, D. E., Wang, M., Brown, E., Fones, L. \& Cave, J. W. Selective repression of gene expression in neuropathic pain by the neuronrestrictive silencing factor/repressor element-1 silencing transcription (NRSF/REST). Neurosci. Lett. 625, 20-25 (2016).

16. Suo, H., Wang, P., Tong, J., Cai, L., Liu, J., Huang, D., Huang, L., Wang, Z., Huang, Y., Xu, J., Ma, Y., Yu, M., Fei, J. \& Huang, F. NRSF is an essential mediator for the neuroprotection of trichostatin A in the MPTP mouse model of Parkinson's disease. Neuropharmacology 99, 67-78 (2015).

17. Katayama, Y., Nishiyama, M., Shoji, H., Ohkawa, Y., Kawamura, A., Sato, T., Suyama, M., Takumi, T., Miyakawa, T. \& Nakayama, K. I. CHD8 haploinsufficiency results in autistic-like phenotypes in mice. Nature 537, 675-679 (2016).

18. Ueda, H., Kurita, J., Neyama, H., Hirao, Y., Kouji, H., Mishina, T., Kasai, M., Nakano, H., Yoshimori, A. \& Nishimura, Y. A mimetic of the mSin3-binding helix of NRSF/REST ameliorates abnormal pain behavior in chronic Pain models. Bioorg. Med. Chem. Lett. 27, 4705-4709 (2017).

19. Ooi, L. \& Wood, I. C. Chromatin crosstalk in development and disease: Lessons from REST. Nat. Rev. Genet. 8, 544-554 (2007).

20. Naruse, Y., Aoki, T., Kojima, T. \& Mori, N. Neural restrictive silencer factor recruits $\mathrm{mSin} 3$ and histone deacetylase complex to repress neuron-specific target genes. Proc. Natl. Acad. Sci. USA 96, 13691-13696 (1999). 
21. Bansal, N., David, G., Farias, E. \& Waxman, S. Emerging roles of epigenetic regulator $\operatorname{Sin} 3$ in cancer. Adv. Cancer Res. 130, 113-135 (2016).

22. Nomura, M., Uda-Tochio, H., Murai, K., Mori, N. \& Nishimura, Y. The neural repressor NRSF/REST binds the PAH1 domain of the $\operatorname{Sin} 3$ corepressor by using its distinct short hydrophobic helix. J. Mol. Biol. 354, 903-915 (2005).

23. Wright, P. E. \& Dyson, H. J. Intrinsically unstructured proteins: Re-assessing the protein structure-function paradigm. J. Mol. Biol. 293, 321-331 (1999).

24. Dyson, H. J. \& Wright, P. E. Intrinsically unstructured proteins and their functions. Nat. Rev. Mol. Cell Biol. 6, 197-208 (2005).

25. Sugase, K., Dyson, H. J. \& Wright, P. E. Mechanism of coupled folding and binding of an intrinsically disordered protein. Nature 447, 1021-1025 (2007).

26. Higo, J., Nishimura, Y. \& Nakamura, H. A Free-energy landscape for coupled folding and binding of an intrinsically disordered protein in explicit solvent from detailed allatom computations. J. Am. Chem. Soc. 133, 10448-10458 (2011).

27. Leone, S., Mutti, C., Kazantsev, A., Sturlese, M., Moro, S., Cattaneo, E., Rigamonti, D. \& Contini, A. SAR and QSAR study on 2-Aminothiazole derivatives, modulators of transcriptional repression in Huntington's disease. Bioorg. Med. Chem. 16, 56955703 (2008).

28. Charbord, J., Poydenot, P., Bonnefond, C., Feyeux, M., Casagrande, F., Brinon, B., Francelle, L., Aurégan, G., Guillermier, M., Cailleret, M., Viegas, P., Nicoleau, C., Martinat, C., Brouillet, E., Cattaneo, E., Peschanski, M., Lechuga, M. \& Perrier, A.L. High throughput screening for inhibitors of REST in neural derivatives of human embryonic stem cells reveals a chemical compound that promotes expression of neuronal genes. Stem Cells 31, 1816-1828 (2013).

29. Conforti, P., Zuccato, C., Gaudenzi, G., Ieraci, A., Camnasio, S., Buckley, N.J., Mutti, C., Cotelli, F., Contini, A. \& Cattaneo, E. Binding of the repressor complex RESTmSIN3b by small molecules restores neuronal gene tanscription in Huntington's disease models. J. Neurochem. 127, 22-35 (2013).

30. Kurita, J., Hirao, Y., Miyata, N. \& Nishimura, Y. NMR screening of mSin3B binding compounds for the interaction inhibition with a neural repressor, NRSF/REST. In Modern Magnetic Resonance, 2nd ed; Webb, G.A., Ed.; Springer: Cham, Switzerland, pp. 705-726 (2017).

31. Kurita, J., Hirao, Y., Nakano, H., Fukunishi, Y. \& Nishimura, Y. Sertraline, chlorprothixene, and chlorpromazine characteristically interact with the RESTbinding site of the corepressor $\mathrm{mSin} 3$, showing medulloblastoma cell growth inhibitory activities. Scientific Reports 8, 13763 (2018). 
32. Hayami, T., Higo, J., Nakamura, H. \& Kasahara, K. Multidimensional virtual-system coupled canonical molecular dynamics to compute free-energy landscapes of peptide multimer assembly. J. Comput. Chem. 40, 2453-2463 (2019).

33. Higo, J., Kasahara, K., Wada, M., Dasgupta, B., Kamiya, N., Hayami, T., Fukuda, I., Fukunishi, Y. \& Nakamura, H. Free-energy landscape of molecular interactions between endothelin 1 and human endothelin type B receptor: Fly-casting mechanism. Protein Eng. Des. Sel. 32, 297-308 (2019).

34. Higo, J., Kusaka, A., Kasahara, K., Kamiya, N., Hayato, I., Qilin, X., Takahashi, T., Fukuda, I., Mori, M., Hata, Y. \& Fukunishi, Y. GA-guided mD-VcMD: A geneticalgorithm-guided method for multi-dimensional virtual-system coupled molecular dynamics. Biophysics and Physicobiology (2020). https://doi.org/10.2142/biophysico.BSJ-2020008

35. Higo, J., Kawabata, T., Kusaka, A., Kasahara, K., Kamiya, N., Fukuda, I., Mori, K., Hata, Y., Fukunishi, Y. \& Nakamura, H. Molecular interaction mechanism of a 14-33 protein with a phosphorylated peptide elucidated by enhanced conformational sampling. J. Chem. Inf. Model. 60, 4867-4880 (2020).

36. Higo, J., Kamiya, N., Sugihara, T., Yonezawa, Y. \& Nakamura, H. Verifying trivial parallelization of multicanonical molecular dynamics for conformational sampling of a polypeptide in explicit water. Chem. Phys. Lett. 473, 326-329 (2009).

37. Ikebe, J., Umezawa, K., Kamiya, N., Sugihara, T., Yonezawa, Y., Takano, Y., Nakamura, H. \& Higo, J. Theory for trivial trajectory parallelization of multicanonical molecular dynamics and application to a polypeptide in water. J. Comput. Chem. 32, 1286-1297 (2011).

38. Kasahara, K., Ma, B., Goto, K., Dasgupta, B., Higo, J., Fukuda, I., Mashimo, T., Akiyama, Y. \& Nakamura, H. myPresto/omegagene: a GPU-accelerated molecular dynamics simulator tailored for enhanced conformational sampling methods with a non-ewald electrostatic scheme. Biophys. Physicobiol. 13, 209-216 (2016).

39. Ryckaert, J.-P., Ciccotti, G. \& Berendsen, H. J. C. Numerical integration of the cartesian equations of motion of a system with constraints: Molecular dynamics of $n$ alkanes. J. Comput. Phys. 23, 327-341 (1977).

40. Berendsen, H. J. C., Postma, J. P. M., van Gunsteren, W. F., DiNola, A. \& Haak, J. R. Molecular dynamics with coupling to an external bath. J. Chem. Phys. 81, 3684 3690 (1984).

41. Kamiya, N., Fukuda, I. \& Nakamura, H. Application of zero-dipole summation method to molecular dynamics simulations of a membrane protein system. Chem. Phys. Lett. 568-569, 26-32 (2013). 
42. Fukuda, I., Kamiya, N., Yonezawa, Y. \& Nakamura, H. Simple and accurate scheme to compute electrostatic interaction: Zero-dipole summation technique for molecular system and application to bulk water. J. Chem. Phys. 137, 054314 (2012).

43. Fukuda, I., Yonezawa, Y. \& Nakamura, H. Molecular dynamics scheme for precise estimation of electrostatic interaction via zero-dipole summation principle. J. Chem. Phys. 134, 164107 (2011).

44. Morishita, T. Fluctuation formulas in molecular-dynamics simulations with the weak coupling heat bath. J. Chem. Phys. 113, 2976-2982 (2000).

45. Kamiya, N., Watanabe, Y. S., Ono, S. \& Higo, J. AMBER-based hybrid force field for conformational sampling of polypeptides. Chem. Phys. Lett. 401, 312-317 (2005).

46. Jorgensen, W. L., Chandrasekhar, J., Madura, J. D., Impey, R. W. \& Klein, M. L. Comparison of simple potential functions for simulating liquid water. J. Chem. Phys. 79, 926-936 (1983).

47. Joung, I. S. \& Cheatham, III, T. E. Determination of alkali and halide monovalent ion parameters for use in explicitly solvated biomolecular simulations. J. Phys. Chem. B 112, 9020-9041 (2008).

48. Frisch, M. J. et al. Gaussian 09, Revision D.01.

49. Bayly, C. I., Cieplak, P., Cornell, W. \& Kollman, P. A. A well-behaved electrostatic potential based method using charge restraints for deriving atomic charges: the RESP model. J. Phys. Chem. 97, 10269-10280 (1993).

50. Wang, J. M., Wolf, R. M., Caldwell, J. W., Kollman, P. A. \& Case, D. A. Development and testing of a general amber force field. J. Comput. Chem. 25, 11571174 (2004).

51. Cornell, W. D., Cieplak, P., Bayly, C. I., Gould, I. R., Merz, K. M., Ferguson, D. M., Spellmeyer, D. C., Fox, T., Caldwell, J. W. \& Kollman, P. A. A second generation force field for the simulation of proteins, nucleic acids, and organic molecules. J. Am. Chem. Soc. 117, 5179-5197 (1995).

52. Kollman, P. A., Dixon, R. W., Cornell, W. D., Chipot, C. \& Pohorille, A. The development/application of a 'minimalist' organic/biochemical molecular mechanic force field using a combination of ab initio calculations and experimental data. In Computer Simulations of Biological Systems; van Gunsteren, W. F., Weiner, P. K. \& Wilkinson, A. J., Eds., Springer, Dordrecht, The Netherlands, 1997; Volume 3, pp. 83-96.

53. Bekker, G., Kawabata, T \& Kurisu G. The Biological Structure Model Archive (BSM-Arc): An Archive for in Silico Models and Simulations. Biophys Rev. 12, 371375 (2020). 


\section{Figure legends}

Figure 1. Density $Q_{\text {cano }}\left(\lambda^{(\alpha)}, \lambda^{(\beta)}, \lambda^{(\gamma)}\right)$ of (a) the sertraline-mSin3B, (b) YN3-mSin3B, and (c) acitretin-mSin3B systems in the $3 \mathrm{D}-\mathrm{RC}$ space. In GA-guided mD-VcMD, the distribution is defined originally by $Q_{\text {cano }}\left(L^{(\alpha)}, L^{(\beta)}, L^{(\gamma)}\right)$, where $L^{(\alpha)}, L^{(\beta)}$, and $L^{(\gamma)}$ are respectively indices to specify the positions $\lambda^{(\alpha)}, \lambda^{(\beta)}$, and $\lambda^{(\gamma)}$ in the $3 \mathrm{D}-\mathrm{RC}$ space. Then, we convert $\left[L_{i}^{(\alpha)}, L_{j}^{(\beta)}, L_{k}^{(\gamma)}\right]$ to: $\lambda_{l}^{(\alpha)}=0.5\left\{\left[\lambda_{i}^{(\alpha)}\right]_{\min }+\left[\lambda_{i}^{(\alpha)}\right]_{\operatorname{mas}}\right\}$, where $i=$ $1, \ldots, n_{v s}(\alpha), \lambda_{j}^{(\beta)}=0.5\left\{\left[\lambda_{j}^{(\beta)}\right]_{\min }+\left[\lambda_{j}^{(\beta)}\right]_{m a s}\right\}$, where $j=1, \ldots, n_{v s}(\beta)$, and $\lambda_{j}^{(\beta)}=$ $0.5\left\{\left[\lambda_{j}^{(\gamma)}\right]_{\min }+\left[\lambda_{j}^{(\gamma)}\right]_{\operatorname{mas}}\right\}, k=1, \ldots, n_{v s}(\gamma)$. See Supplementary Table S3 for values of $\left[\lambda_{i}^{(h)}\right]_{\min },\left[\lambda_{i}^{(h)}\right]_{\max }$, and $n_{v s}(h)(h=\alpha, \beta, \gamma)$. Then, $Q_{\text {cano }}\left(\lambda^{(\alpha)}, \lambda^{(\beta)}, \lambda^{(\gamma)}\right)$ is normalized so that the highest density is set to 1 . Contour levels are presented by colors in inset.

Figure 2. Spatial density $\rho_{C G}^{(s)}(\boldsymbol{r})$ of the geometric center (GC) at position $\boldsymbol{r}$ for (a) the sertraline-mSin3B, (b) YN3-mSin3B, and (c) acitretin-mSin3B systems in the 3D real space. See Supplementary Section 4 for procedure to calculate $\rho_{C G}^{(s)}(\boldsymbol{r})$. Contour levels are shown in inset where $\rho_{0}=0.001$. Displayed structure of mSin3B is that after NPT simulation for each system, where labels $\mathrm{H} 2$ and H3 represent helix 2 and helix 3, respectively. The high-density cluster $\left(\rho_{C G}^{(s)}(\boldsymbol{r})>0.5 \rho_{0}\right)$ in the cleft of mSin3B (PAH1) is named as Cluster A, and one near the N-terminal of mSin3B as Cluster B. (d) NMR structure of REST/NRSF-mSin3B complex (PDB ID: 2CZY), where cyan-colored model is REST/NRSF, and the magenta-colored segment is the LIML sequence of REST/NRSF. Label $\mathrm{C}$ indicates the position of the $\mathrm{C}$-terminal tail of $\mathrm{mSin} 3 \mathrm{~B}$. Two magenta-colored side-chains are Leu 46 and Leu 49 of the LIML sequence. Black broken-line circle indicates the position of the two sidechains. Those circles in panels (a), (b), and (c) are presented to indicate the sidechain position of Leu 46 and Leu 49. Green-colored sidechains are Val 75, Phe 93, and Phe 96 of mSin3B (see also green-colored sidechains of Supplementary Figure S12a), which form a hydrophobic core with Leu 46 and Leu 49 of the LIML sequence. 
Figure 3. Radial distribution functions (RDFs) $p\left(r_{R}^{(s)}\right)$ for (a) the sertraline-mSin3B, (b) YN3-mSin3B, and (c) acitretin-mSin3B systems. Three RDFs $p\left(r_{\text {Val75 }}^{(s)}\right), p\left(r_{P h e 93}^{(s)}\right)$, and $p\left(r_{\text {Phe96 }}^{(s)}\right)$ are shown by different colors as indicated in inset of panel (a). See Supplementary Section 6 for procedure to calculate RDF.

Figure 4. Spatial density $\rho_{C G A}^{(s)}(\boldsymbol{r})$ (blue-colored contours) for the geometric center of Ring A of sertraline in the sertraline-mSin3B system, where the contour level is $\rho_{C G A}^{(s)}(\boldsymbol{r})=0.5 \rho_{0}\left(\rho_{0}=0.001\right)$. Chemical structure of sertraline is also shown. Redcolored contours are $\rho_{C G}^{(s)}(\boldsymbol{r})$ for the geometric center of the entire sertraline. Labels H2 and $\mathrm{H} 3$ represent helices 2 and 3, respectively. Green-colored residues are Val 75, Phe 93, and Phe 96 of $\mathrm{mSin} 3 \mathrm{~B}$. See Supplementary Section 4 for procedure to calculate $\rho_{C G A}^{(s)}(\boldsymbol{r})$.

Figure 5. (a)-(d) Sertraline-mSin $3 B$ complexes picked randomly from regions of $\rho_{C G A}^{(s)}(\boldsymbol{r})>0.5 \rho_{0}\left(\rho_{0}=0.001\right)$, which are shown by blue-colored contours. Magentacolored portion is Ring A of Sertraline. Red-colored vectors are those pointing from Ring$\mathrm{BC}$ geometrical center to that of Ring A (see Supplementary Figure S1b for positions of Ring A and BC). Green-colored residues are Val 75, Phe 93, and Phe 96 of mSin3B. Labels $\mathrm{H} 1, \ldots, \mathrm{H} 4$ are helices $1-4$ of $\mathrm{mSin} 3 \mathrm{~B}$ (PAH1 domain).

Figure 6. Spatial pattern of $\left\langle\boldsymbol{e}^{(s)}(\boldsymbol{r})\right\rangle$ for sertraline in the cleft of mSin3B viewed from two different directions. Red and black vectors are those with $\left|\left\langle\boldsymbol{e}^{(s)}(\boldsymbol{r})\right\rangle\right| \geq$ 0.5 and $\left|<\boldsymbol{e}^{(s)}(\boldsymbol{r})>\right|<0.5$, respectively. Gray contours represent the high-density regions of $\rho_{C G}^{(s)}(\boldsymbol{r})>0.5 \rho_{0} \quad\left(\rho_{0}=0.001\right)$. The vectors $\left\langle\boldsymbol{e}^{(s)}(\boldsymbol{r})\right\rangle$ are shown in regions with $\rho_{C G}^{(s)}(\boldsymbol{r})>0.5 \rho_{0}$. This figure also displays REST/NRSF bound to mSin3B, and the magenta-colored segment is the LIML sequence of REST/NRSF. Note that REST/NRSF is not involved in the current simulation. The shown structure is the REST/NRSF-mSin3B complex structure (PDB ID: 2CZY). Labels H1,.., H4 are helices $1, \ldots, 4$ of $\mathrm{mSin} 3 \mathrm{~B}$ (PAH1 domain).

Figure 7. Spatial pattern of $\left\langle\boldsymbol{e}^{(s)}(\boldsymbol{r})>\right.$ for $\mathrm{YN} 3$ in the cleft of mSin3B viewed from two different directions. Gray contours represent the high-density regions of $\rho_{C G}^{(s)}(\boldsymbol{r})>$ $0.5 \rho_{0}\left(\rho_{0}=0.001\right)$. See caption of Figure 6 for more information. 
Figure 8. Spatial pattern of $\left\langle\boldsymbol{e}^{(s)}(\boldsymbol{r})\right\rangle$ for acitretin in the cleft of $\mathrm{mSin} 3 \mathrm{~B}$ viewed from two different directions. Gray contours represent the high-density regions of $\rho_{C G}^{(s)}(\boldsymbol{r})>$ $0.1 \rho_{0}\left(\rho_{0}=0.001\right)$. See caption of Figure 6 for more information.

Figure 9. (a) Structures of the three compounds sertraline, YN3, and acitretin. For each compound, inter-atomic distance $r_{\text {atom-pair }}^{(s)}(s=$ sertraline, YN3, or acitretin) is defined between cyan- to orange-colored atoms. A cyan-colored atom, which is involved in a ring for each ligand, is not set on the ring rotation axis to detect the ring-rotational motions. (b) Distance distribution functions (DDFs) $P\left(r_{\text {atom-pair }}^{(s)}\right)$ for the three distances. See Supplementary Section 6 for procedure to calculate DDF.

Figure 10. (a) Chemical structures of mSin3B binders, and their orientation aligned from head to tail sides. Rings A, B and C of sertraline are depicted in magenta, black, and orange, respectively. Blue circles represent aromatic rings that are expected to interact with the hydrophobic cleft of $\mathrm{mSin} 3 \mathrm{~B}$ from conventional structure-activity relationship (SAR). Green circles represent $\pi$-electron rich regions. (b) Presence/absence of Medulloblastoma cell-growth inhibition activities for the compounds. (c) The actual orientations of the compounds with respect to the cleft of $\mathrm{mSin} 3 \mathrm{~B}$, predicted by the present MD simulation study.

Figure 11. Scheme of a compound that binds to the cleft of $\mathrm{mSin} 3 \mathrm{~B}$. 
Figures

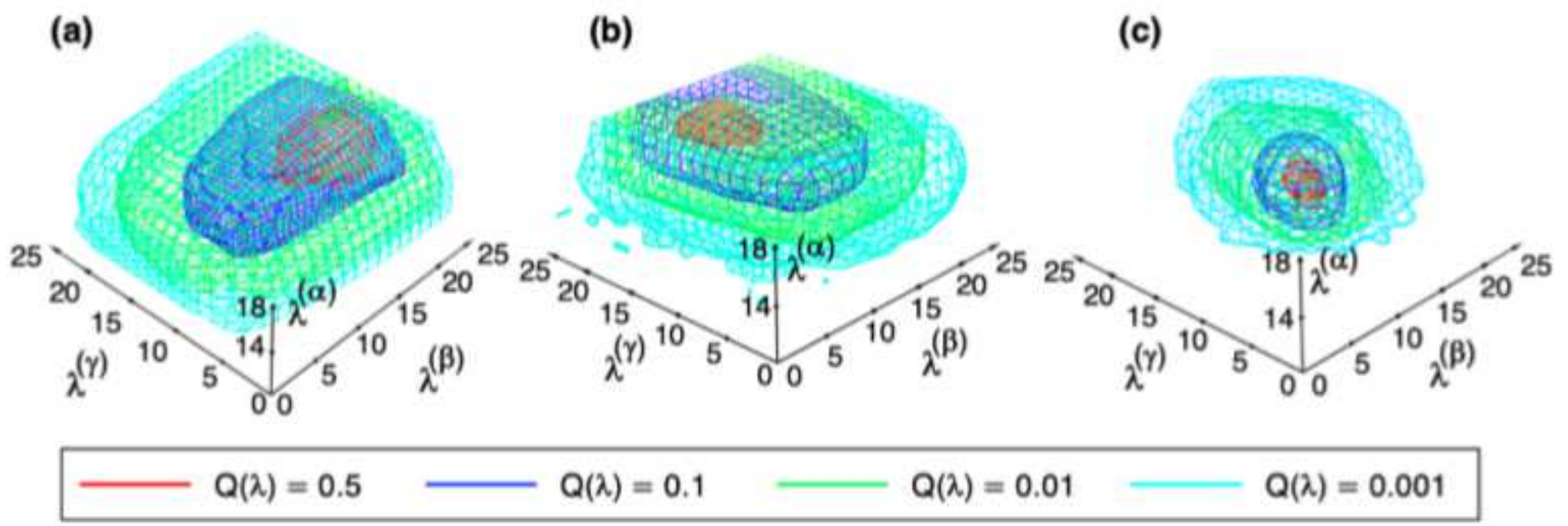

Figure 1

"See the Supplemental Files section for the complete figure caption".

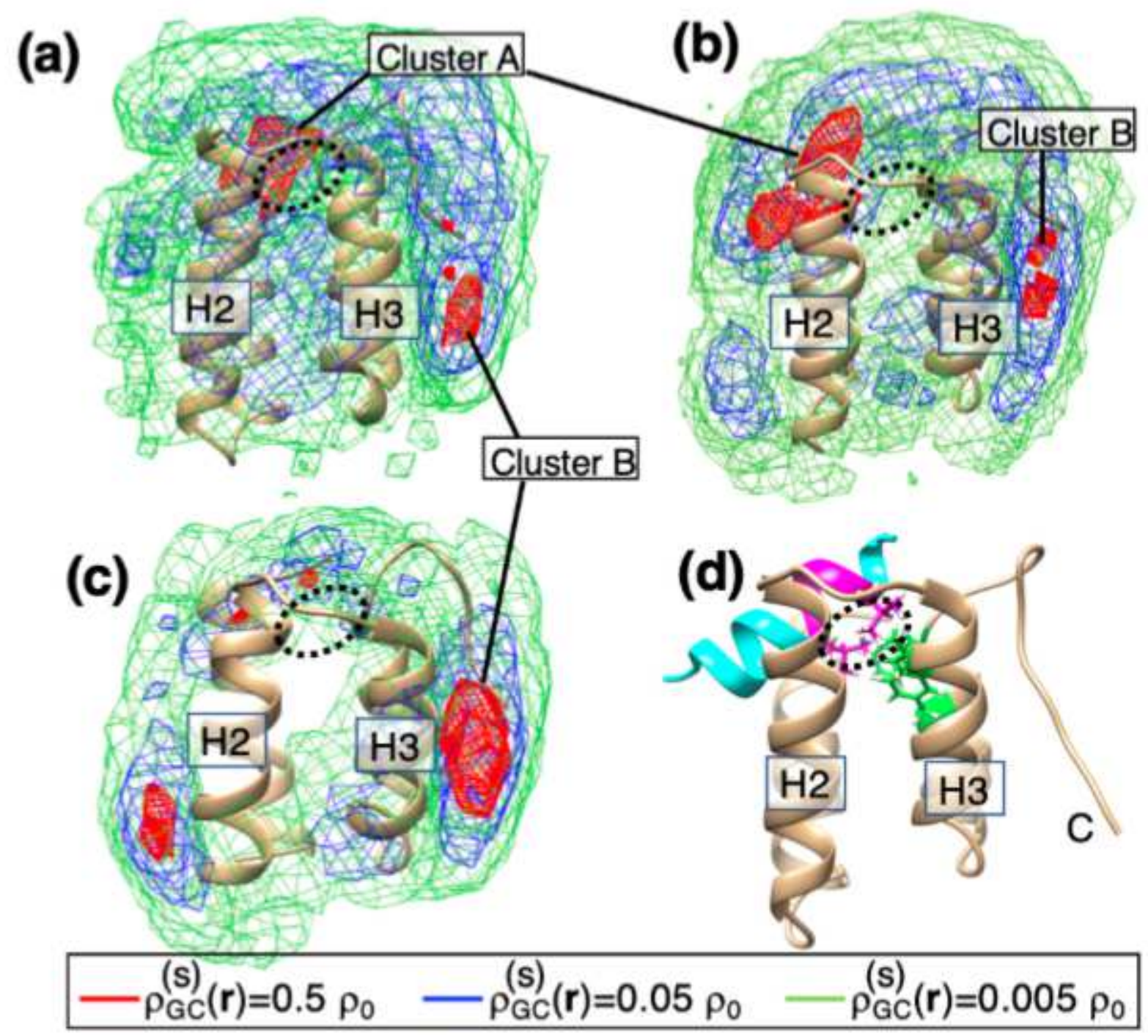


Figure 2

"See the Supplemental Files section for the complete figure caption".
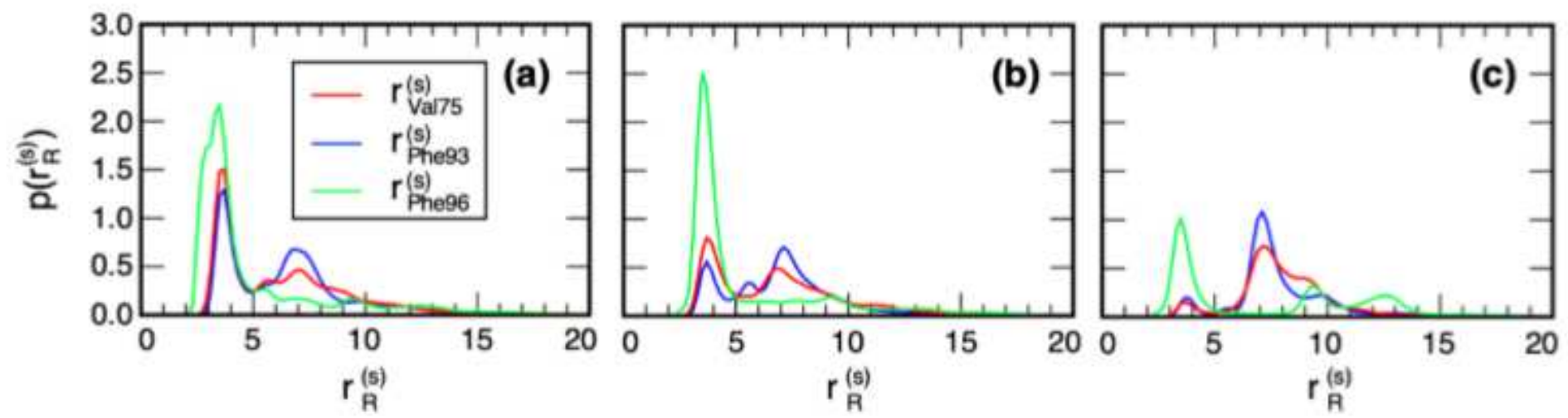

Figure 3

"See the Supplemental Files section for the complete figure caption".

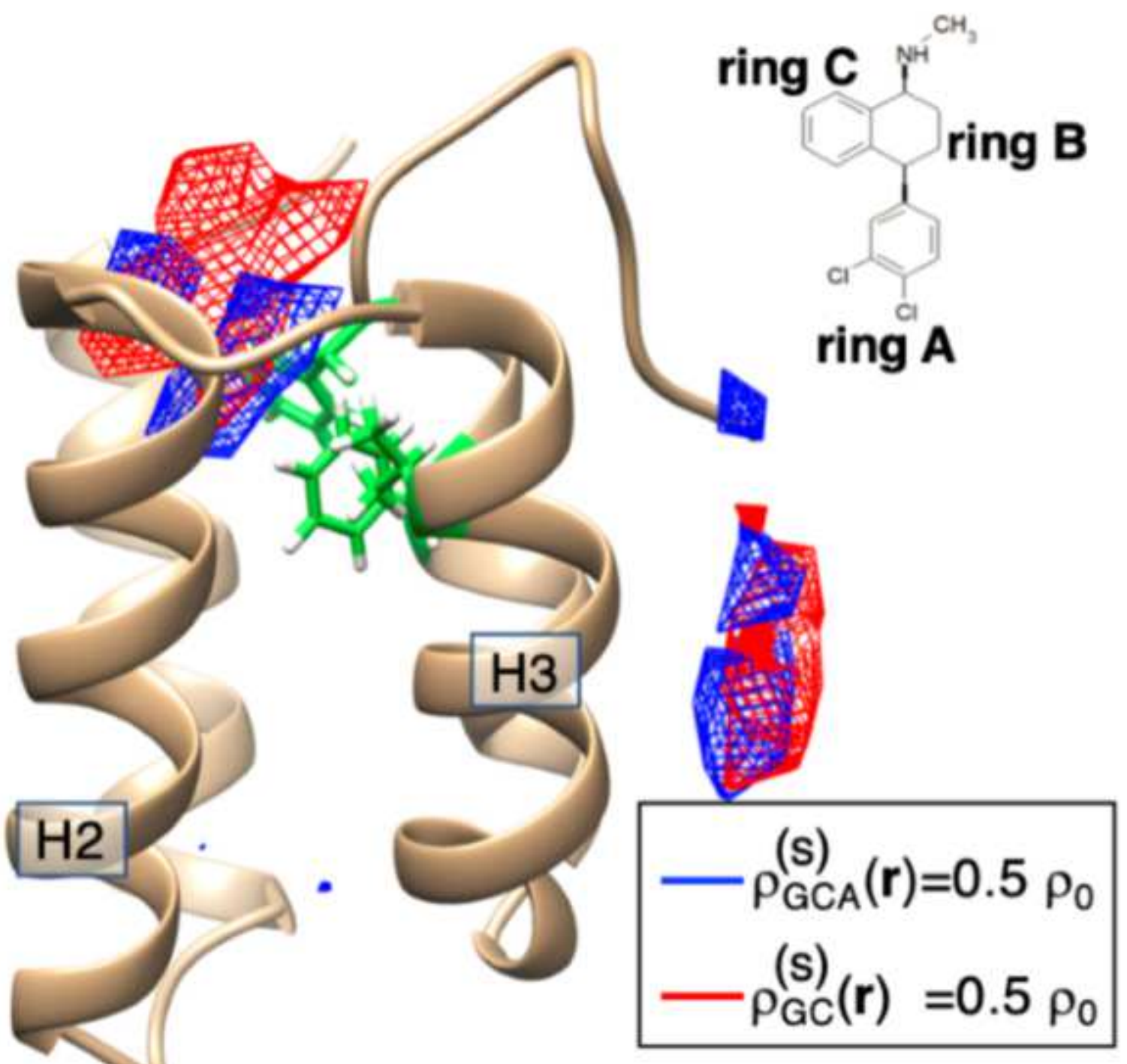


Figure 4

"See the Supplemental Files section for the complete figure caption".

(a)

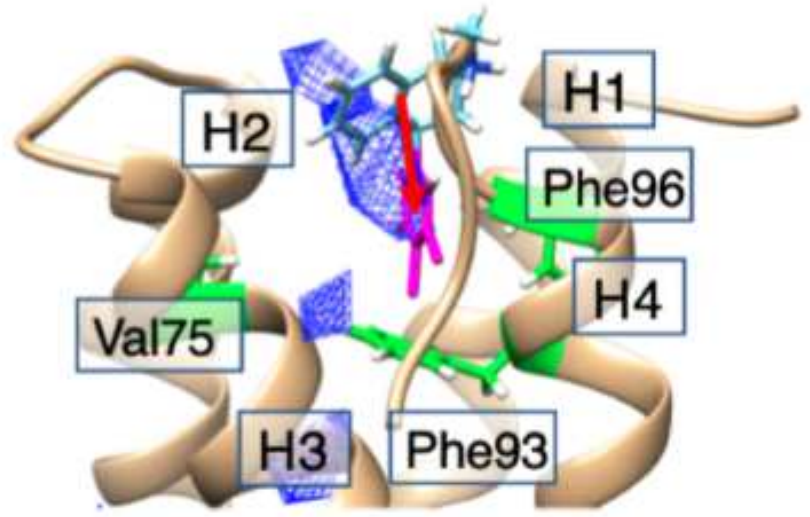

(c)

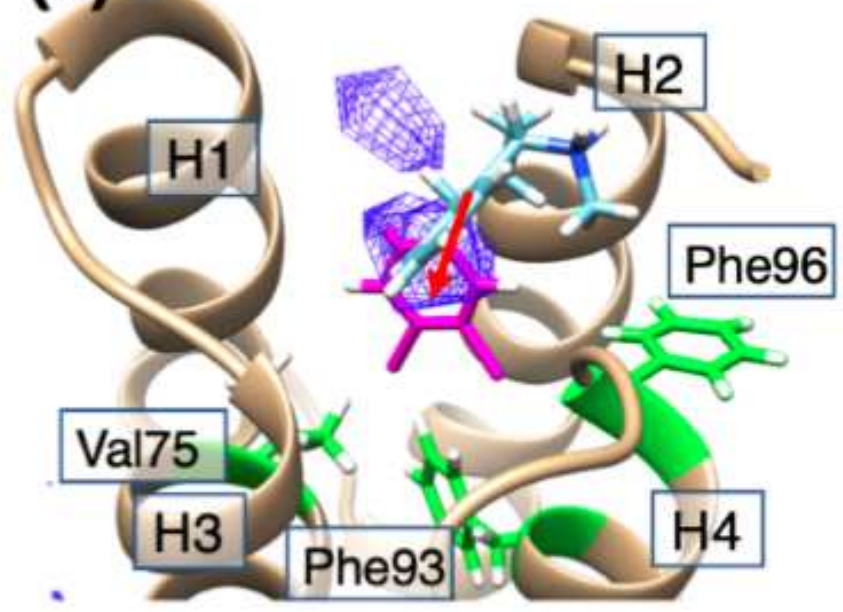

(b)

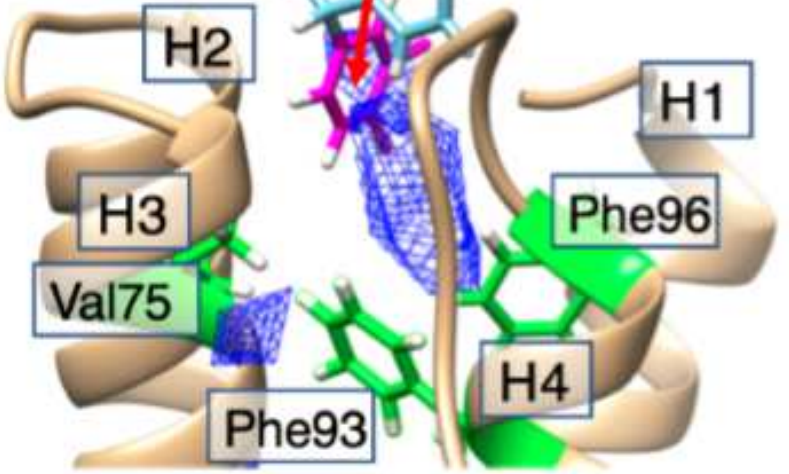

(d)

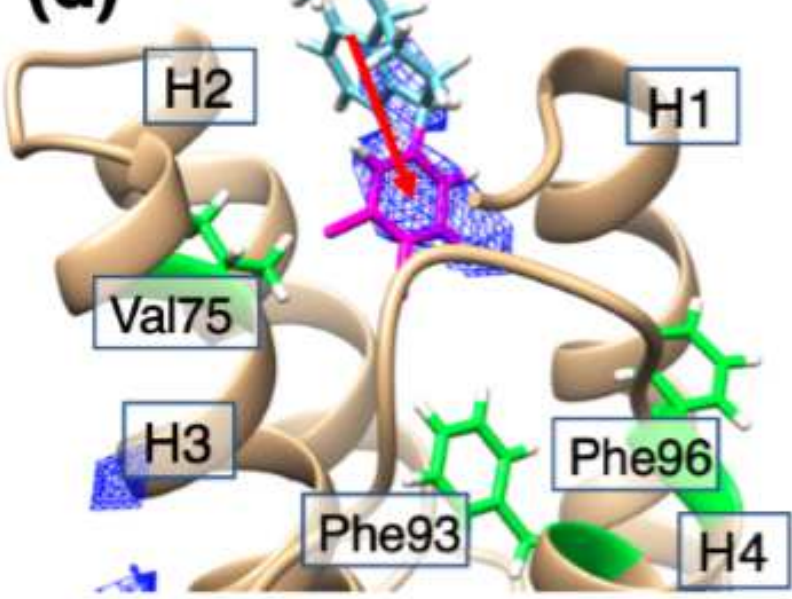

Figure 5

"See the Supplemental Files section for the complete figure caption".
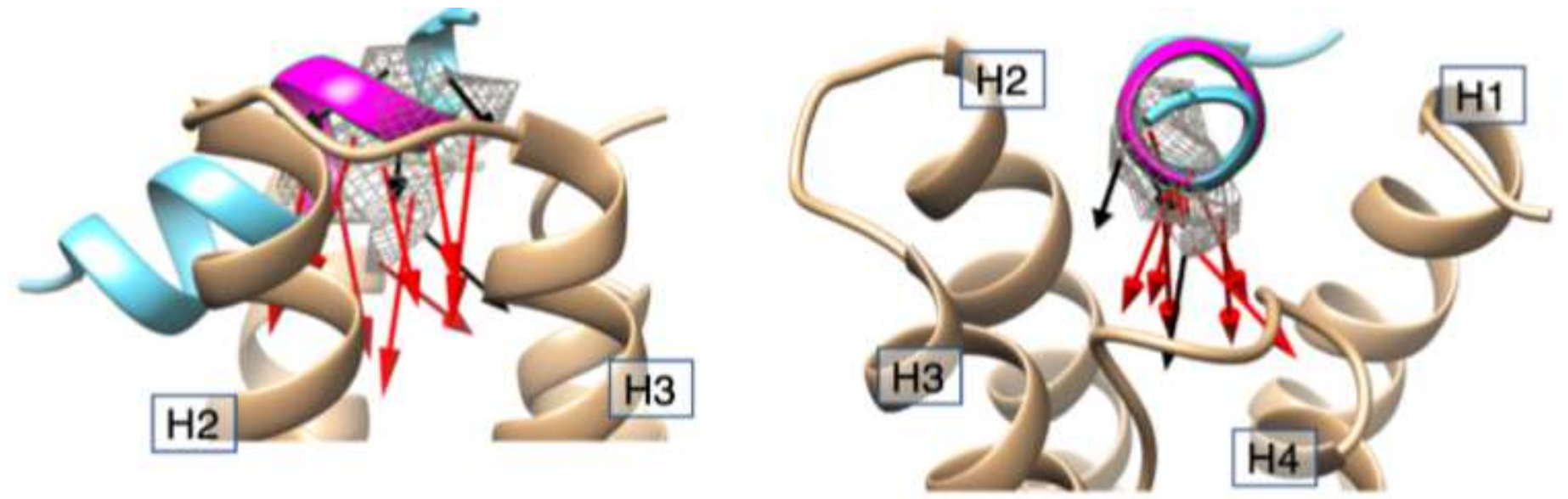

Figure 6 
"See the Supplemental Files section for the complete figure caption".
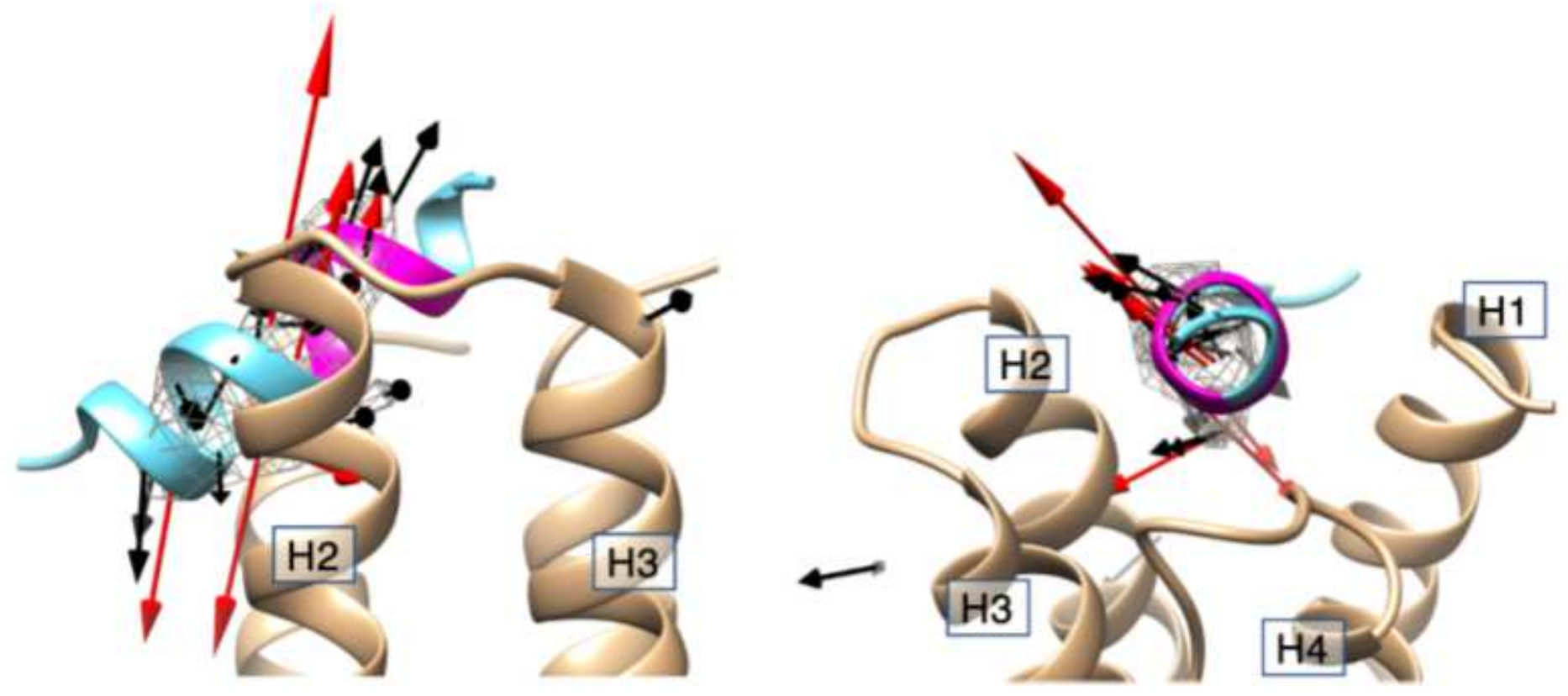

Figure 7

"See the Supplemental Files section for the complete figure caption".
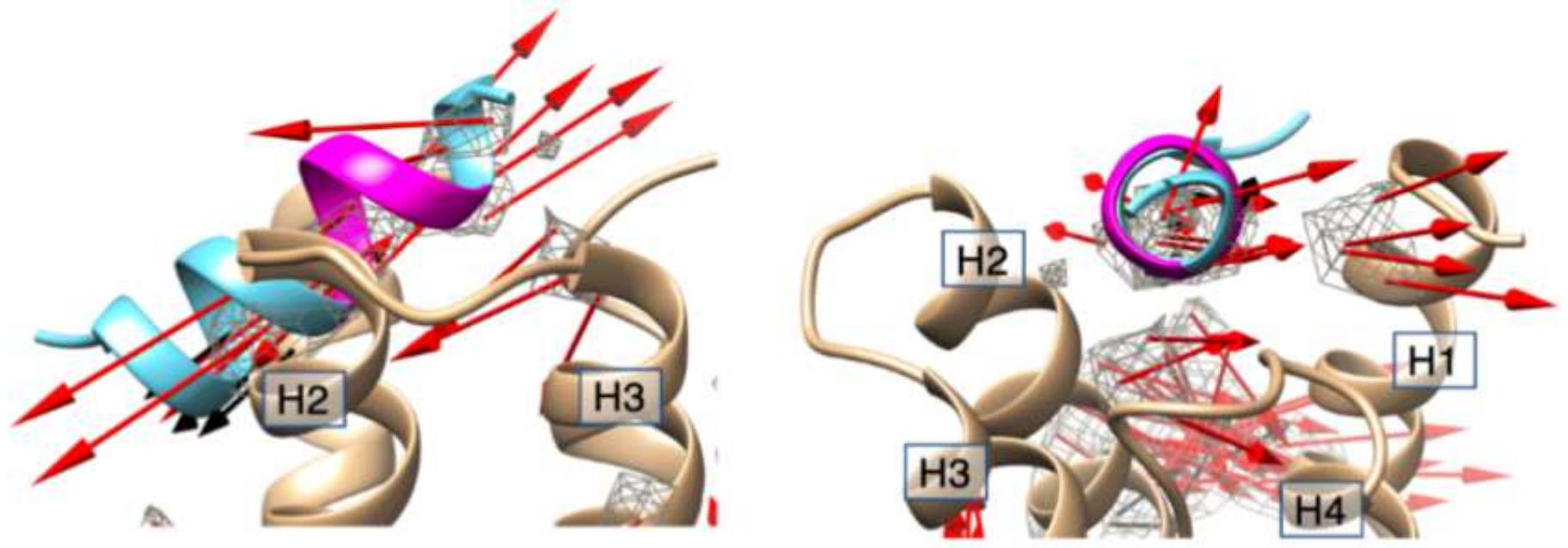

Figure 8

"See the Supplemental Files section for the complete figure caption". 
(a)

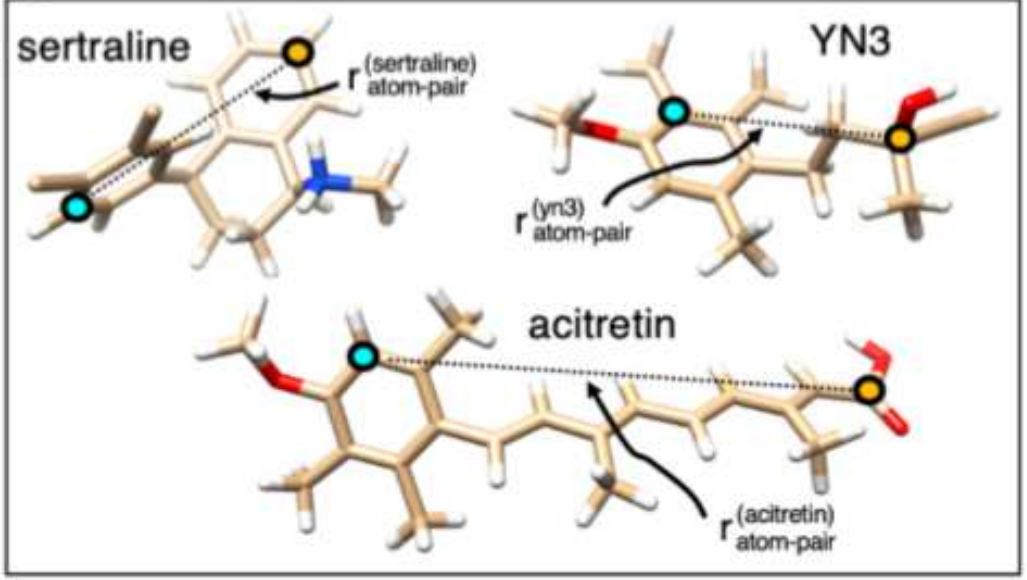

(b)

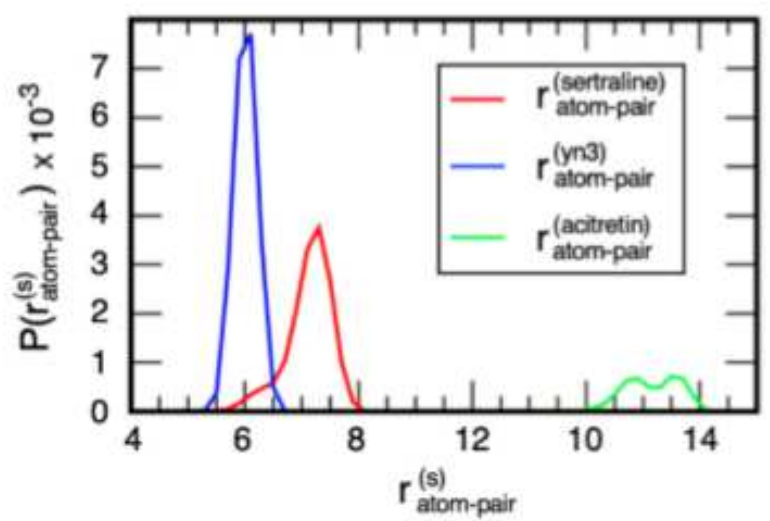

Figure 9

"See the Supplemental Files section for the complete figure caption".

Sertraline

(a)

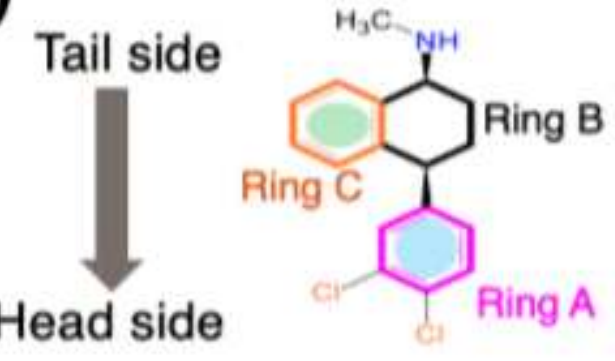

YN3

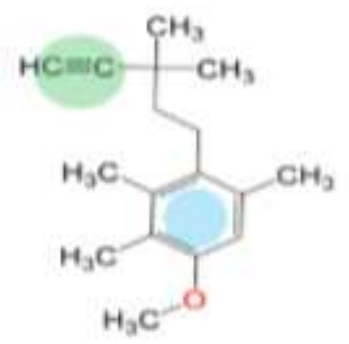

Acitretin

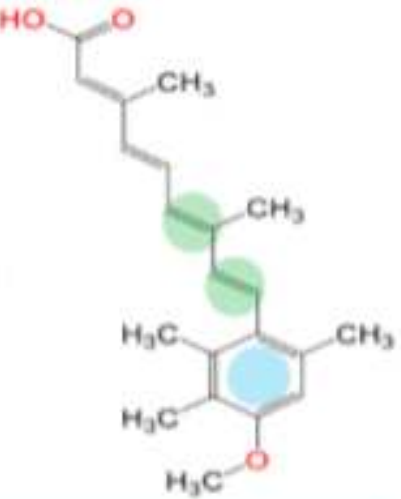

(b)

\begin{tabular}{|ccc|}
\hline \multicolumn{3}{|c|}{ Medulloblastoma cell growth inhibition } \\
\hline Active & Inactive & Inactive \\
\hline
\end{tabular}

(c)

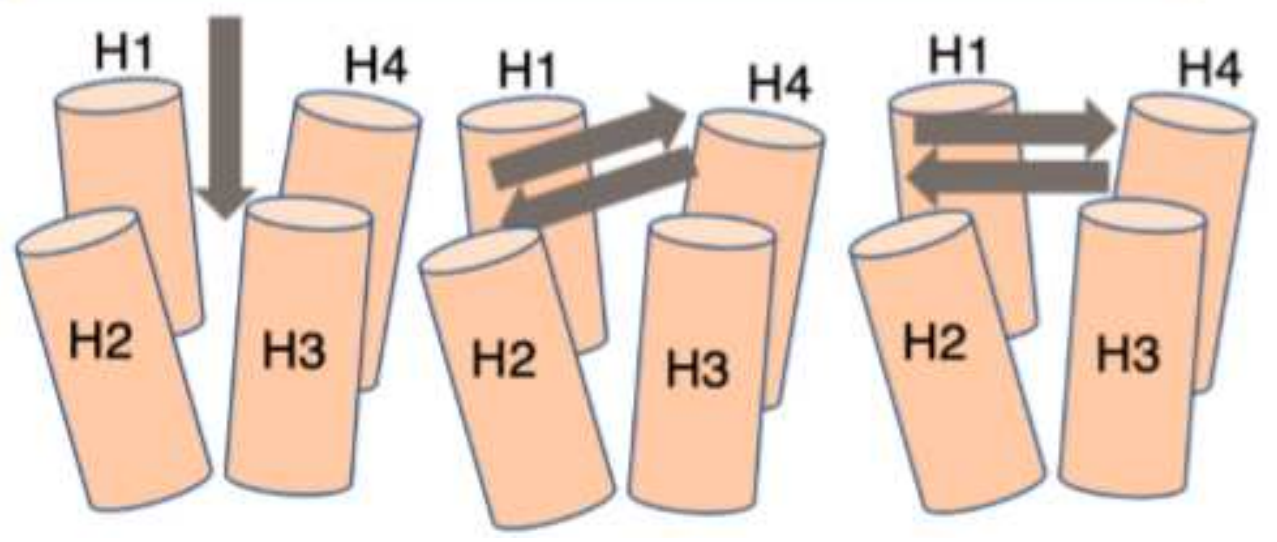

Figure 10

"See the Supplemental Files section for the complete figure caption". 


\section{framework}

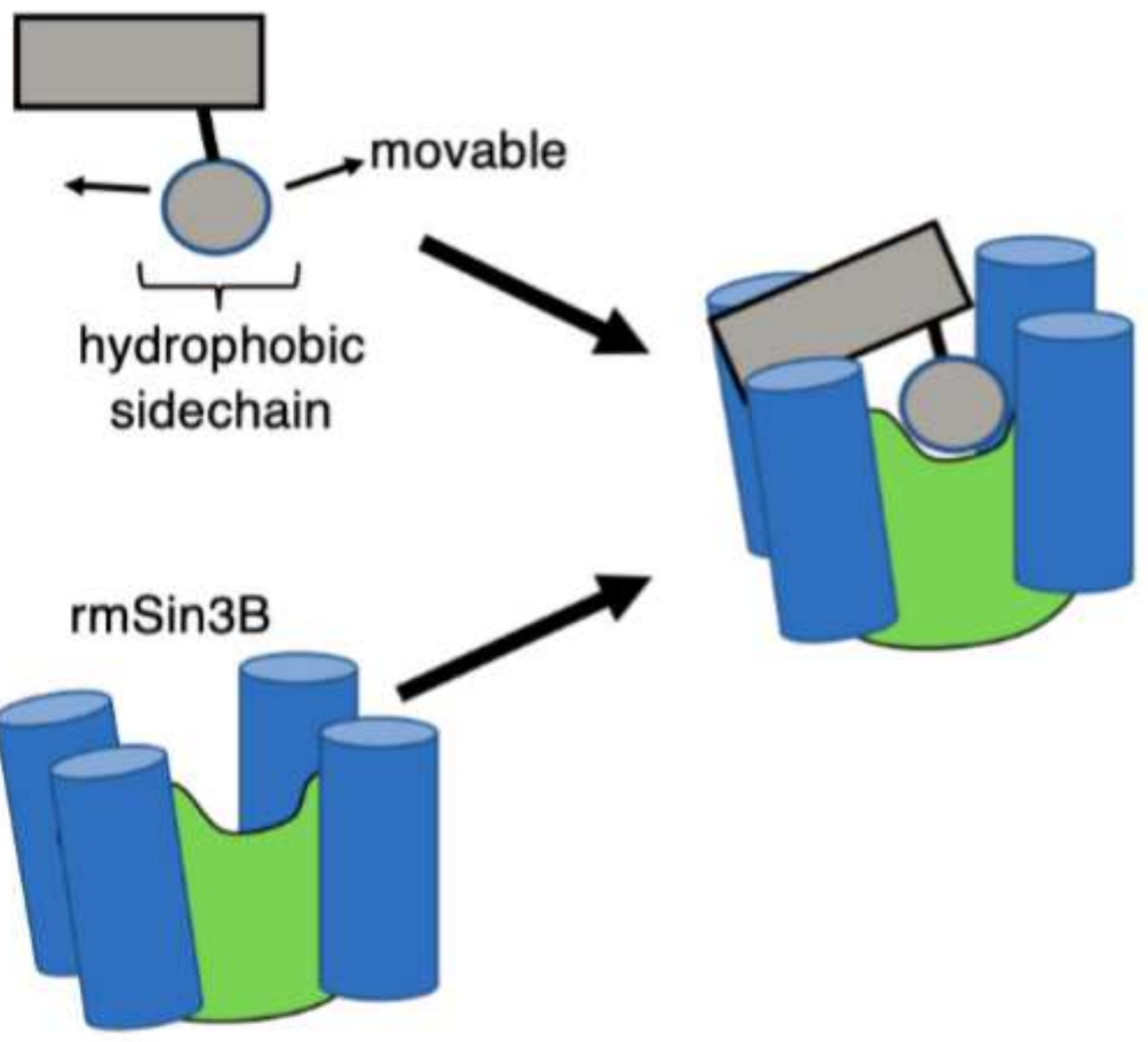

Figure 11

Scheme of a compound that binds to the cleft of mSin3B.

\section{Supplementary Files}

This is a list of supplementary files associated with this preprint. Click to download.

- mSin3B3compSISciRep.pdf

- FigureLegend.pdf 